\title{
Synthesis, Structure, and Properties of Helical Bis-Cu(II) Complex of Linear Hexapyrrolic Ligand
}

Sabari Panchavarnam, Poornenth Pushpanandan and Mangalampalli Ravikanth*

Indian Institute of Technology, Powai, Mumbai, 400076, India, Fax:91-22-5723480;

Tel: 91-22-5767176; E-mail: ravikanth@chem.iitb.ac.in

\section{Table of contents}

\begin{tabular}{|c|l|c|}
\hline Sl. no & \multicolumn{1}{|c|}{ Details } & Page no. \\
\hline 1 & General experimental section & S2-S3 \\
\hline 2 & Figures S1-S6. Characterization (HRMS and NMR) data for all new compounds & S4-S9 \\
\hline 3 & Figure S7-S8. Dihedral angle of compounds 2 and 1-Cu & S12-S21 \\
\hline 4 & Tables S1-S5. X-ray crystal data for the compound 2 and 1-Cu & S22 \\
\hline 5 & Figure S9. EPR spectra of compound 1-Cu & S24 \\
\hline 6 & Figure S10. Comparison of CV compounds 2 and 1-Cu & S28-S39 \\
\hline 7 & Figure S11-S13. Molecular orbital diagram of compound 2 and 1-Cu & S40-S44 \\
\hline 8 & Tables S6-S7. Td-DFT transition data of Compound 2 and 1-Cu & S45 \\
\hline 10 & Tables S8-S9. Cartesian coordinates of the optimized (S0) geometries of the compounds 2 and 1-Cu. & \\
\hline
\end{tabular}




\section{General Experimental section}

\section{Materials, methods, instrumentation and computational details.}

Important chemicals such as Boron trifluoride-diethyl ether complex $\left(\mathrm{BF}_{3} . \mathrm{OEt}_{2}\right)$ and 2,3-dichloro-5,6-dicyano-1,4-benzoquinone (DDQ), diisopropylethylamine (DIPEA), Copper (II) chloride salts were obtained from Aldrich. Silica (60-120 mesh) used for column chromatography was obtained from Acme Chemicals, India. Commercially available solvents and reagents were used without further purification unless otherwise mentioned or dry solvent was necessary. Chloroform (HPLC grade) from Merck India, was used for all the absorption and fluorescence measurements, and dichloromethane (HPLC) for cyclic voltammetry (CV) analysis without further purification. Deuterated chloroform $\left(\mathrm{CDCl}_{3}\right)$, was purchased from Sigma-Aldrich, USA and used for NMR studies. The ${ }^{1} \mathrm{H}$ and ${ }^{13} \mathrm{C}$ NMR spectra were recorded in $\mathrm{CDCl}_{3}$ on $\mathrm{Bruker}^{400}$ and $500 \mathrm{MHz}$ instruments. Tetramethylsilane $\left[\mathrm{Si}\left(\mathrm{CH}_{3}\right)_{4}\right]$ was used as an internal standard for ${ }^{1} \mathrm{H}$ and ${ }^{13} \mathrm{C}$ NMR. Absorption spectra were obtained with a Shimadzu UV-Vis spectrophotometer. The high resolution mass spectra were recorded with a Q-TOF micro mass spectrometer. The Singlecrystal X-ray structure analyses were carried out on a Rigaku Saturn724 diffractometer conjugated with a low-temperature attachment. Data were collected at $100 \mathrm{~K}$ by means of graphite monochromated Mo Ka radiation $\left(\lambda_{\alpha}=0.71073 \AA\right)$ by the $\omega$-scan method. The structures were solved by direct methods and refined by least-squares against F2 employing the software packages SHELXL-97,18 SIR-92,19 and WINGX.20. All nonhydrogen atoms were refined anisotropically. The CCDC No. for compound $\mathbf{2}$ and 1-Cu are 2110848, and 2110847, and the X-Ray crystallographic data of these compounds are given in supporting information. These data can be obtained free of charge from The Cambridge Crystallographic Data Centre via www.ccdc.cam.ac.uk/data_request/cif. ESR spectra of 1-Cu was recorded in Bruker EMX Plus series equipped with temperature controller. A Gaussian 09 program package was used for performing all the calculations. The initial structures were based on the X-ray crystal 
structures of $\mathbf{2}$ and 1-Cu. The optimized structures of compounds $\mathbf{2}$ was generated using Becke's three-parameter hybrid functional combined with the Lee-Yang-Parr correlation functional or B3LYP level of DFT, with basis set 6-31G(d,p) and for 1-Cu hybrid functional uB3LYP and with basis set $6-31 G(d, p)$ was used ${ }^{1-4}$. Identical basis and functional hybrid sets were used to obtain the oscillator strengths, and vertical excitation energies were obtained for $\mathrm{S}_{0} \rightarrow \mathrm{Sn}$ transitions by the help of TD-DFT techniques ${ }^{5-9}$. 

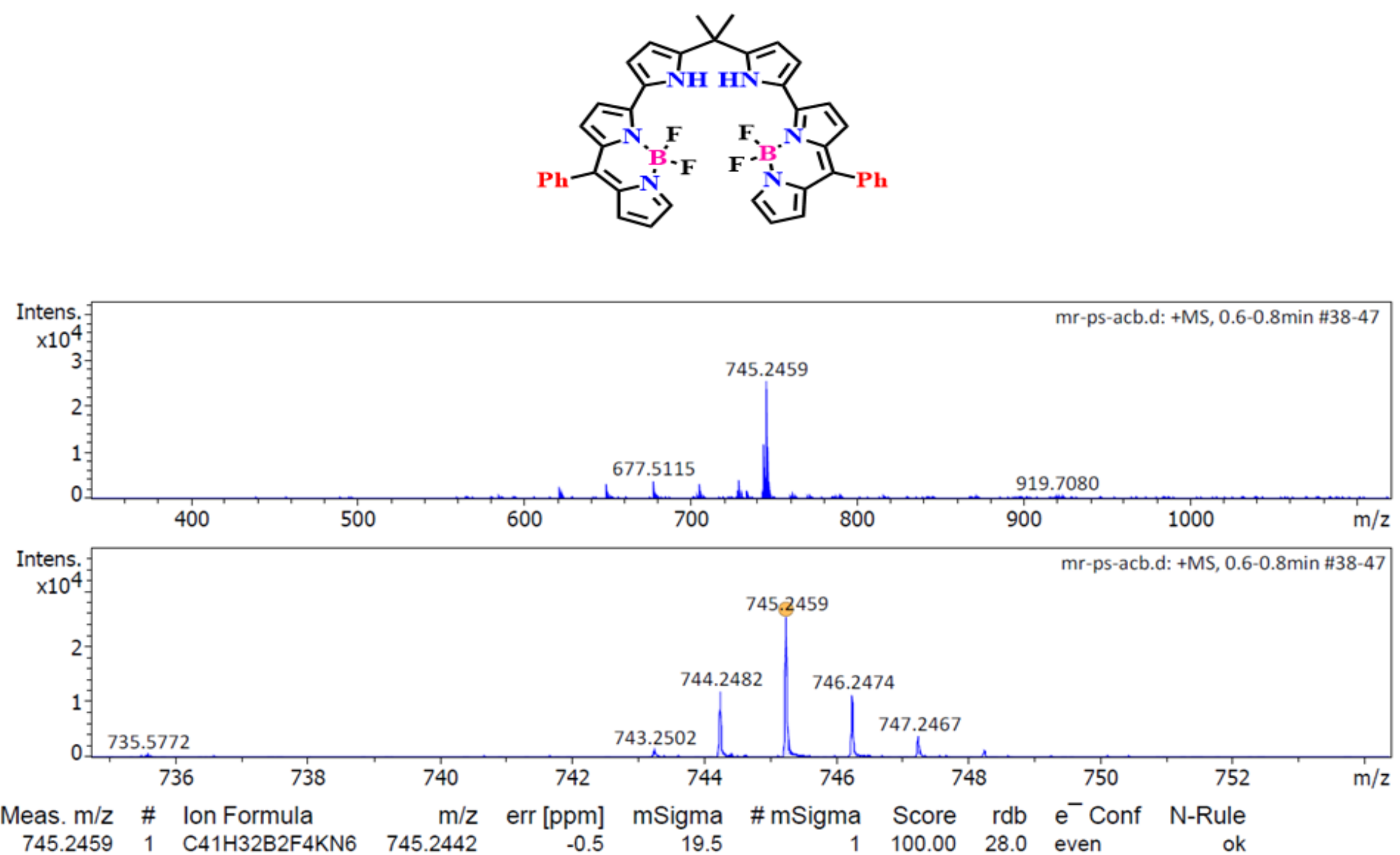

Figure S1: High resolution mass spectra of compound 2. 

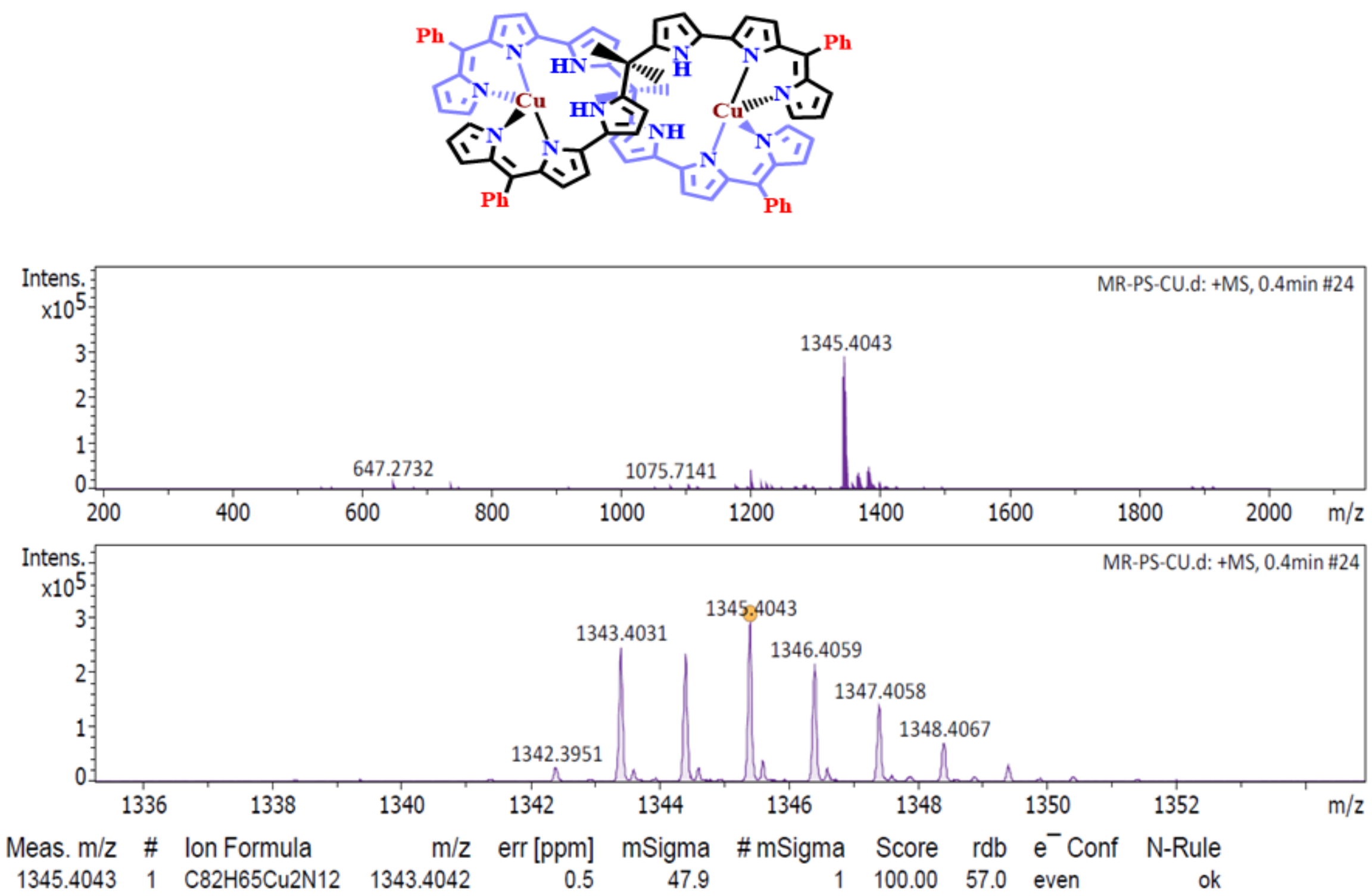

Figure S2: High resolution mass spectra of Compound 1-Cu. 

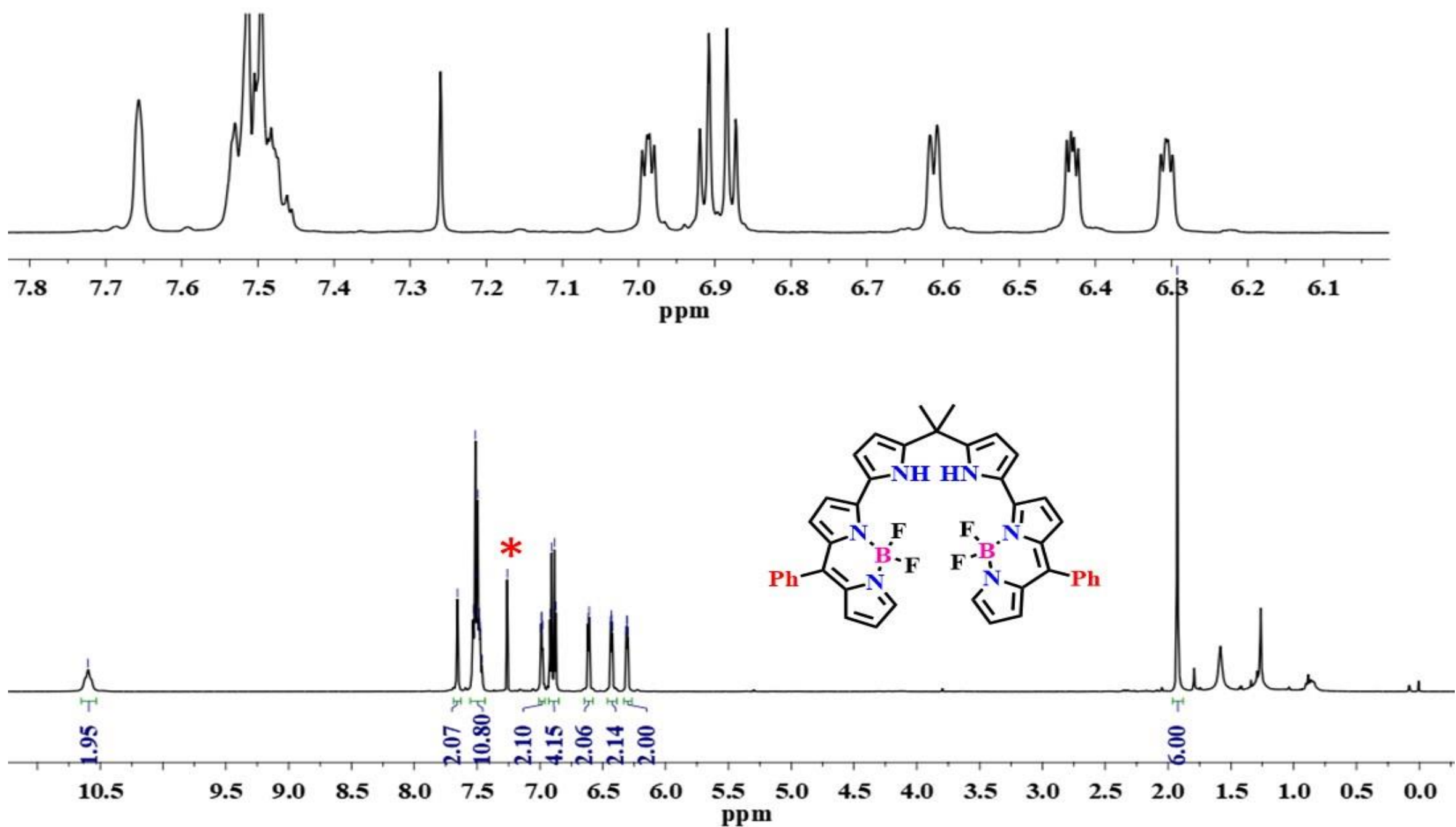

Figure S3: ${ }^{1} \mathrm{H}$ NMR spectrum of compound 2 in $\mathrm{CDCl}_{3}$. The inset shows the expansion.* indicates residual solvent peak S6 

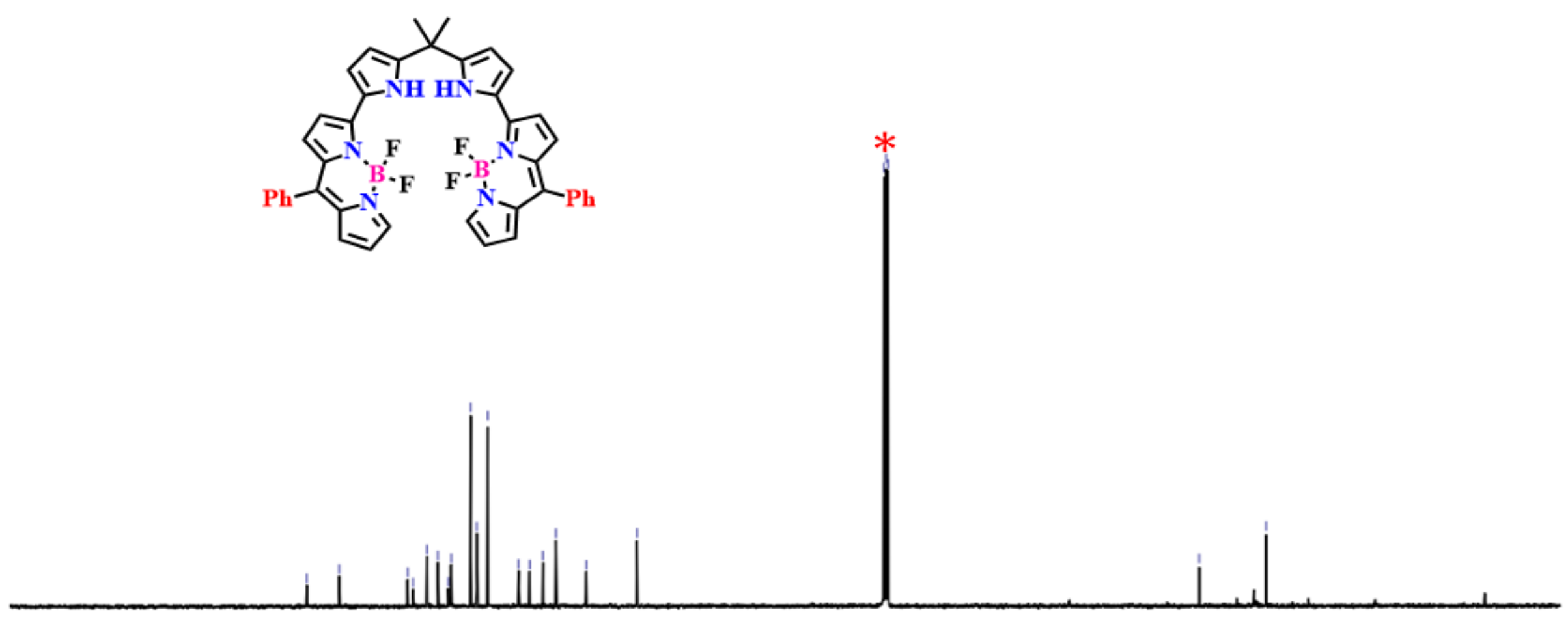

180

Figure S4: ${ }^{13} \mathrm{C}$ NMR spectrum of compound 2 in $\mathrm{CDCl}_{3}$ * indicates residual solvent peak 

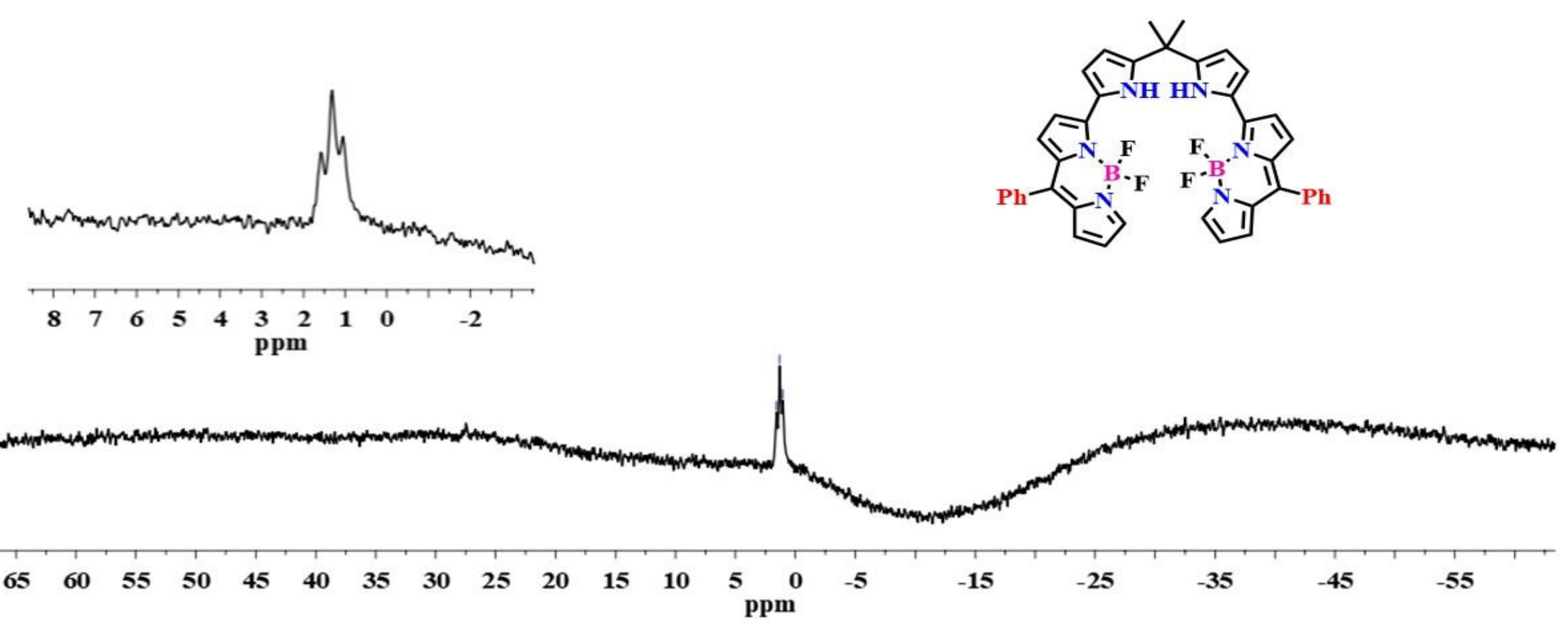

Figure S5: ${ }^{11} \mathrm{~B}$ NMR spectrum of compound 2 in $\mathrm{CDCl}_{3}$. The inset shows the expansion. 

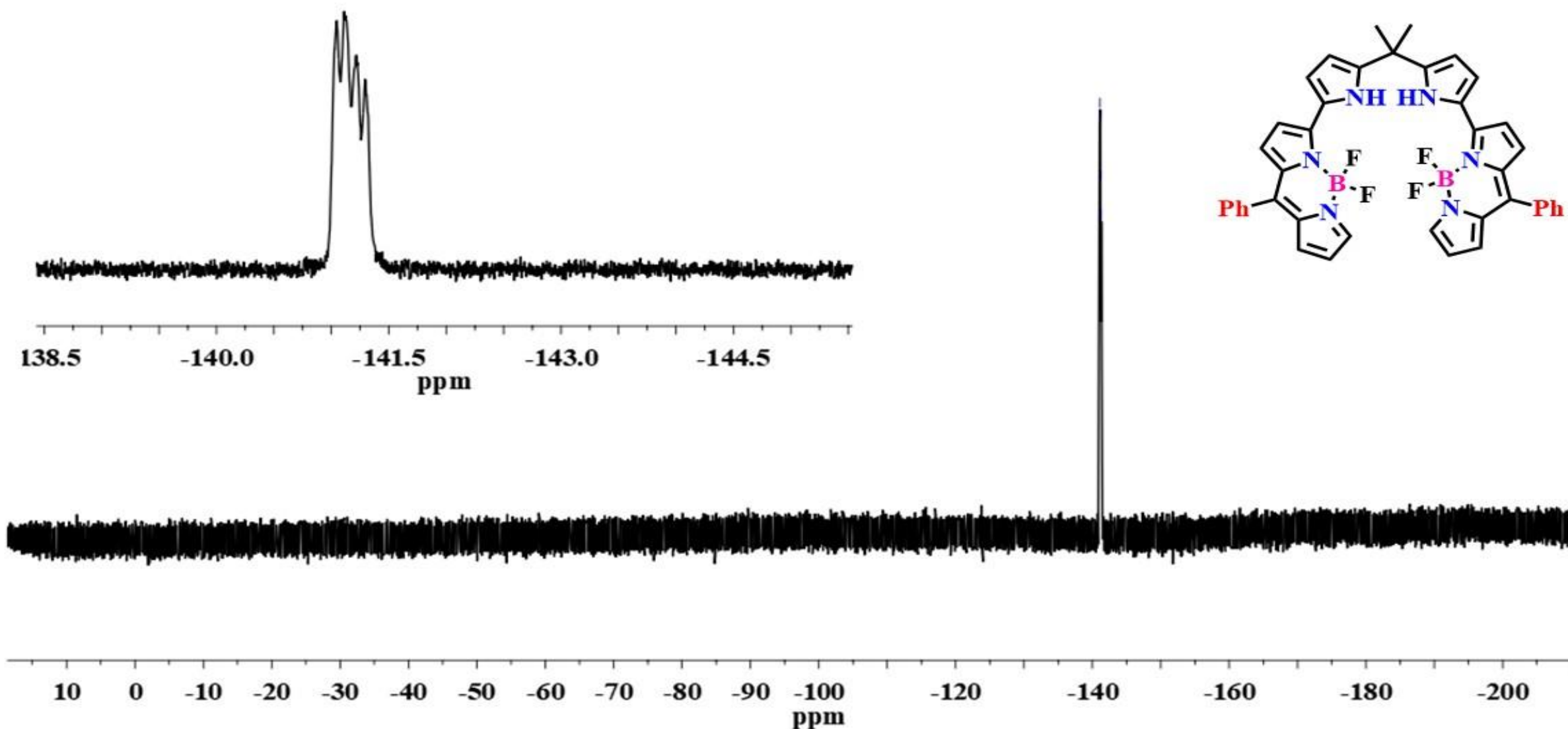

$-120$

$-140$

$-160$

$-\mathbf{1 8 0}$

$-200$

Figure S6: ${ }^{19} \mathrm{~F}$ NMR spectrum of compound 2 in $\mathrm{CDCl}_{3}$. The inset shows the expansion. 


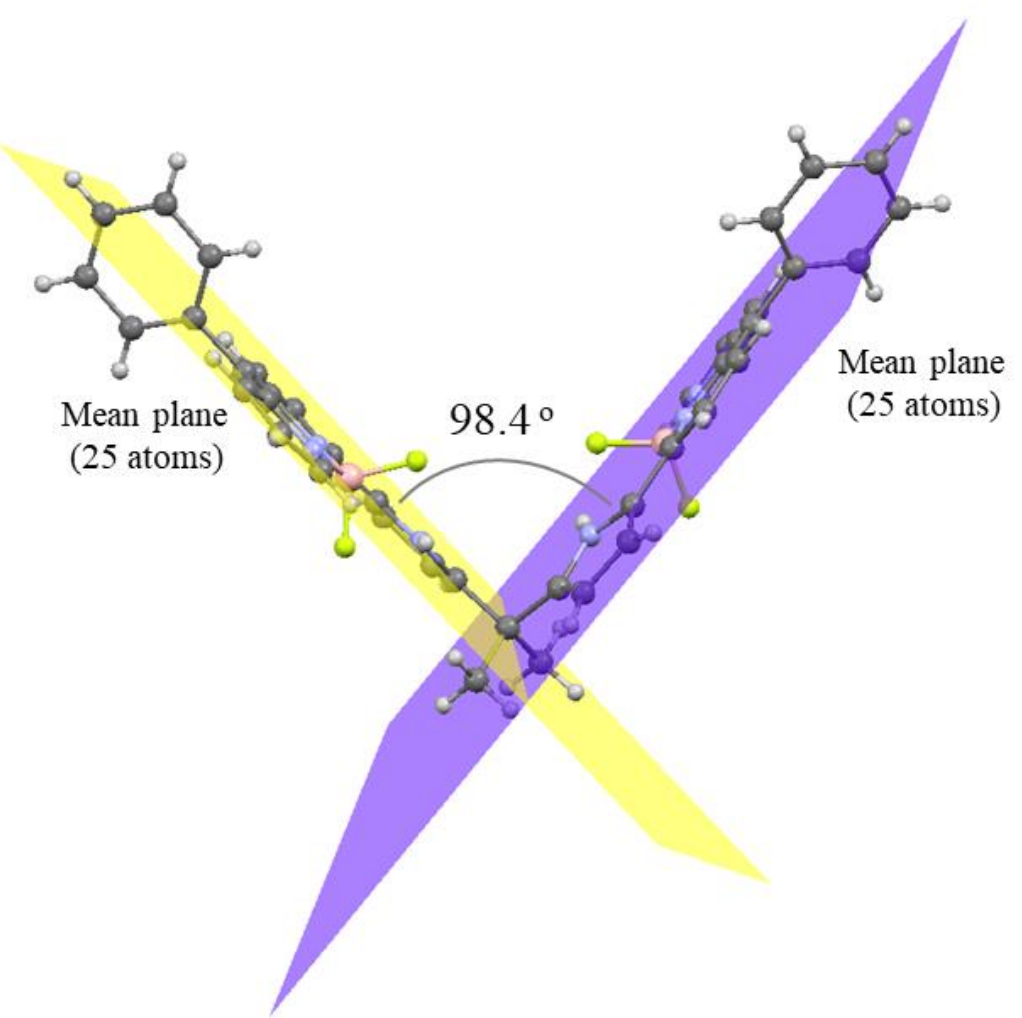

Figure S7: Dihedral angle of bis-BODIPY compound 2 calculated considering two mean planes containing 25 atoms each 


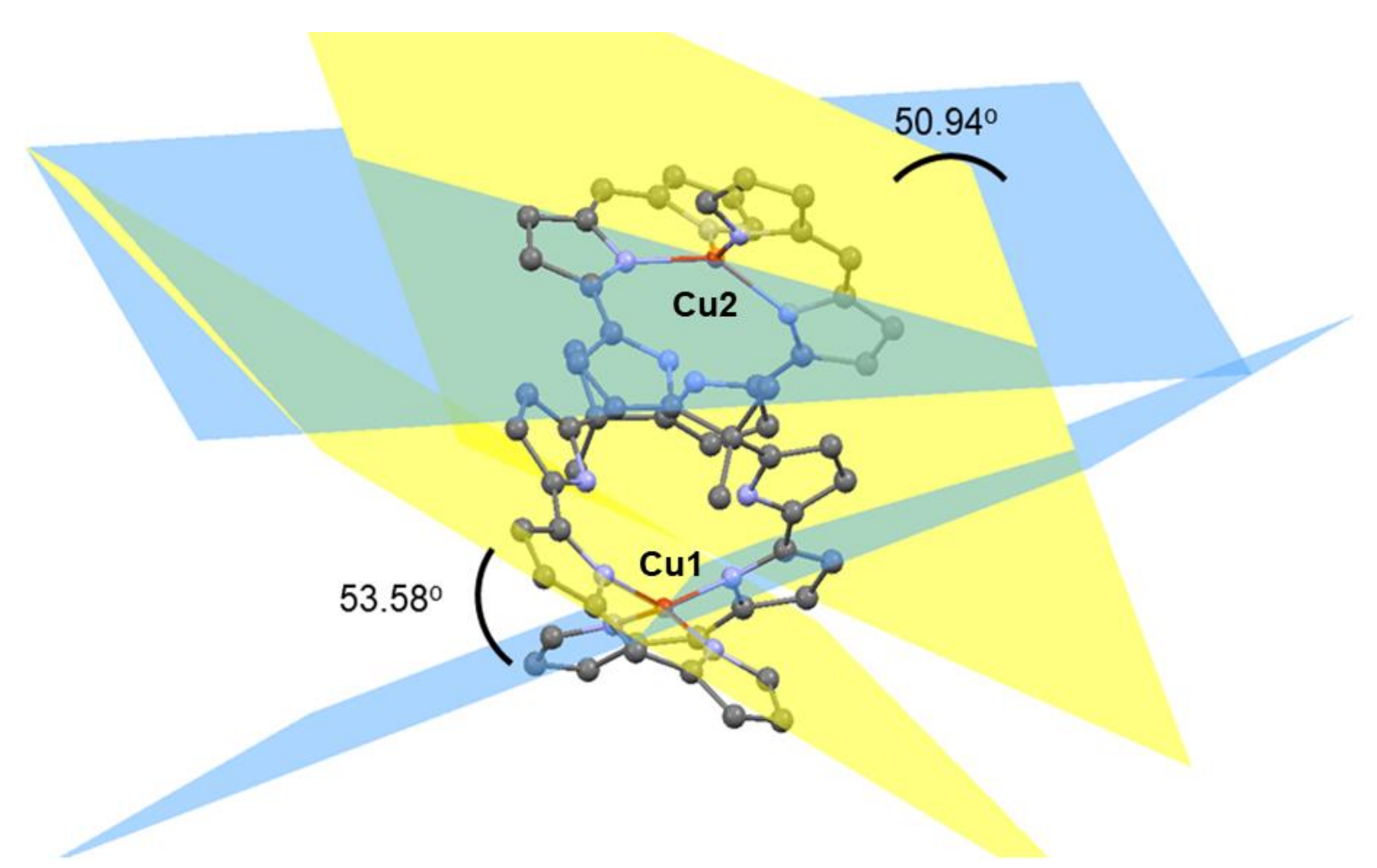

Figure S8: Dihedral angle between N1-Cu-N2 (Yellow) and N3-Cu-N4 (blue) planes formed for Cu1 and Cu2 
Table S1: Crystal data and structure refinement for Compound 2 and 1-Cu

\begin{tabular}{|c|c|c|}
\hline Compound & 2 & 1-Cu \\
\hline Empirical Formula & $\mathrm{C}_{42} \mathrm{H}_{33} \mathrm{~B}_{2} \mathrm{Cl}_{3} \mathrm{~F}_{4} \mathrm{~N}_{6}$ & $\mathrm{C}_{82} \mathrm{H}_{64} \mathrm{Cu}_{2} \mathrm{~N}_{12}$ \\
\hline Crystal System & monoclinic & monoclinic \\
\hline Space Group & $\mathrm{P} 2{ }_{1} / \mathrm{n}$ & $\mathrm{P} 2{ }_{1} / \mathrm{c}$ \\
\hline$R_{1}(I>2.00 \sigma(I))$ & 0.0607 & 0.0602 \\
\hline$w R_{2}$ (All reflections) & 0.1539 & 0.1355 \\
\hline GOF & 1.029 & 1.004 \\
\hline$a[\AA]$ & $9.8732(5)$ & $13.106(2)$ \\
\hline$b[\AA]$ & $24.2220(11)$ & $24.022(3)$ \\
\hline$c[\AA]$ & $16.0491(7)$ & $21.006(2)$ \\
\hline$\alpha\left[^{\circ}\right]$ & 90 & 90 \\
\hline$\beta\left[^{\circ}\right]$ & $95.117(4)$ & $96.553(7)$ \\
\hline$\gamma\left[^{\circ}\right]$ & 90 & 90 \\
\hline$V\left[\AA^{3}\right]$ & $3822.8(3)$ & $6570.2(15)$ \\
\hline$Z$ & 4 & 4 \\
\hline$T[\mathrm{~K}]$ & 100 & 200 \\
\hline$D_{\text {calc }}\left[\mathrm{g} / \mathrm{cm}^{3}\right]$ & 1.435 & 1.359 \\
\hline$F_{000}$ & 1698.5 & 2792.0 \\
\hline $2 \theta_{\max }\left[{ }^{\circ}\right]$ & 50 & 50.08 \\
\hline no. reflns measd. & 14005 & 11575 \\
\hline
\end{tabular}




\begin{tabular}{|c|c|c|}
\hline (unique) & & \\
\hline no. params. & 516 & 865 \\
\hline CCDC no. & 2110848 & 2110847 \\
\hline
\end{tabular}


Table S2: Bond Lengths for compound 2.

\begin{tabular}{|c|c|c|c|c|c|}
\hline Atom & Atom & Length/A & Atom & Atom & Length/Å \\
\hline Cl1 & C42 & 1.749 ( 4 ) & C6 & C5 & $1.493(4)$ \\
\hline $\mathrm{Cl} 2$ & $\mathrm{C} 42$ & $1.771(4)$ & C6 & $\mathrm{C} 7$ & $1.400(5)$ \\
\hline $\mathrm{Cl3}$ & $\mathrm{C} 42$ & $1.764(4)$ & C6 & $\mathrm{C} 11$ & $1.378(4)$ \\
\hline F3 & B2 & $1.413(4)$ & $\mathrm{C} 23$ & $\mathrm{C} 24$ & $1.385(5)$ \\
\hline F2 & B1 & $1.407(4)$ & $\mathrm{C} 30$ & $\mathrm{C} 31$ & $1.381(5)$ \\
\hline F4 & B2 & $1.390(4)$ & $\mathrm{C} 30$ & C29 & $1.421(5)$ \\
\hline F1 & B1 & $1.399(4)$ & C19 & $\mathrm{C} 18$ & $1.379(5)$ \\
\hline N3 & C19 & $1.360(4)$ & $\mathrm{C} 5$ & $\mathrm{C} 12$ & $1.379(5)$ \\
\hline N3 & C16 & $1.374(4)$ & $\mathrm{C} 16$ & $\mathrm{C} 17$ & $1.387(5)$ \\
\hline N2 & $\mathrm{C} 15$ & $1.365(4)$ & $\mathrm{C} 3$ & $\mathrm{C} 2$ & $1.395(5)$ \\
\hline $\mathbf{N} 2$ & $\mathrm{C} 12$ & $1.414(4)$ & $\mathrm{C} 1$ & $\mathrm{C} 2$ & $1.393(5)$ \\
\hline $\mathbf{N} 2$ & B1 & $1.553(5)$ & $\mathrm{C} 26$ & $\mathrm{C} 27$ & $1.426(5)$ \\
\hline N5 & $\mathrm{C} 30$ & $1.405(4)$ & $\mathrm{C} 26$ & $\mathrm{C} 25$ & $1.394(5)$ \\
\hline N5 & $\mathrm{C} 27$ & $1.364(4)$ & C39 & $\mathrm{C} 38$ & $1.399(5)$ \\
\hline N5 & B2 & $1.564(5)$ & C39 & $\mathrm{C} 40$ & $1.401(5)$ \\
\hline N1 & $\mathrm{C} 4$ & $1.397(4)$ & C38 & C31 & $1.421(5)$ \\
\hline N1 & $\mathrm{C} 1$ & $1.367(4)$ & $\mathrm{C} 40$ & $\mathrm{C} 41$ & $1.391(5)$ \\
\hline N1 & B1 & $1.533(5)$ & C33 & $\mathrm{C} 32$ & $1.406(5)$ \\
\hline N4 & $\mathrm{C} 23$ & $1.367(4)$ & C33 & C34 & $1.391(5)$ \\
\hline N4 & $\mathrm{C} 26$ & $1.385(4)$ & $\mathrm{C} 7$ & $\mathrm{C} 8$ & $1.386(5)$ \\
\hline N6 & $\mathrm{C} 38$ & $1.394(4)$ & C31 & $\mathrm{C} 32$ & $1.489(4)$ \\
\hline N6 & $\mathrm{C} 41$ & $1.357(5)$ & $\mathrm{C} 27$ & $\mathrm{C} 28$ & $1.437(5)$ \\
\hline N6 & B2 & $1.529(5)$ & C9 & $\mathrm{C} 8$ & $1.381(5)$ \\
\hline $\mathrm{C4}$ & $\mathrm{C} 5$ & $1.412(5)$ & $\mathrm{C} 9$ & $\mathrm{C} 10$ & $1.377(5)$ \\
\hline $\mathrm{C4}$ & $\mathrm{C} 3$ & $1.404(5)$ & $\mathrm{C} 17$ & $\mathrm{C} 18$ & $1.408(5)$ \\
\hline C13 & C14 & $1.356(5)$ & $\mathrm{C} 24$ & $\mathrm{C} 25$ & $1.400(5)$ \\
\hline C13 & C12 & $1.420(5)$ & $\mathrm{C} 32$ & C37 & $1.404(5)$ \\
\hline C15 & C14 & $1.429(4)$ & C34 & $\mathrm{C} 35$ & $1.381(5)$ \\
\hline C15 & C16 & $1.428(4)$ & C37 & $\mathrm{C} 36$ & $1.397(5)$ \\
\hline C20 & $\mathrm{C} 23$ & $1.514(5)$ & C29 & $\mathrm{C} 28$ & $1.356(5)$ \\
\hline C20 & C19 & $1.516(4)$ & C36 & C35 & $1.383(6)$ \\
\hline
\end{tabular}




\begin{tabular}{llllll}
\hline $\mathbf{C 2 0}$ & $\mathrm{C} 21$ & $1.540(5)$ & C11 & C10 & $1.394(5)$ \\
\hline $\mathbf{C 2 0}$ & C22 & $1.543(5)$ & & \\
\hline
\end{tabular}

Table S3: Bond Angles for compound 2

\begin{tabular}{|c|c|c|c|c|c|c|c|}
\hline Atom & Atom & Atom & Angle ${ }^{\circ}$ & Atom & Atom & Atom & Angle/ ${ }^{\circ}$ \\
\hline C19 & N3 & C16 & $110.6(3)$ & $\mathrm{C} 1$ & $\mathrm{C} 2$ & $\mathrm{C} 3$ & $107.2(3)$ \\
\hline C15 & N2 & $\mathrm{C} 12$ & $107.6(3)$ & N4 & $\mathrm{C} 26$ & $\mathrm{C} 27$ & $126.3(3)$ \\
\hline C15 & $\mathrm{N} 2$ & B1 & $129.5(3)$ & N4 & $\mathrm{C} 26$ & $\mathrm{C} 25$ & $106.2(3)$ \\
\hline C12 & N2 & B1 & $122.7(3)$ & $\mathrm{C} 25$ & $\mathrm{C} 26$ & $\mathrm{C} 27$ & $127.5(3)$ \\
\hline C30 & N5 & B2 & $123.1(3)$ & C38 & C39 & $\mathrm{C} 40$ & $107.0(3)$ \\
\hline C27 & N5 & $\mathrm{C} 30$ & $108.2(3)$ & N6 & C38 & C39 & $108.6(3)$ \\
\hline C27 & N5 & B2 & $128.4(3)$ & N6 & C38 & C31 & $119.6(3)$ \\
\hline $\mathrm{C4}$ & N1 & B1 & $126.1(3)$ & C39 & C38 & C31 & $131.8(3)$ \\
\hline C1 & N1 & $\mathrm{C} 4$ & $107.2(3)$ & C41 & $\mathrm{C} 40$ & C39 & $106.9(4)$ \\
\hline C1 & N1 & B1 & $126.7(3)$ & C34 & C33 & C32 & $120.2(4)$ \\
\hline C23 & N4 & $\mathrm{C} 26$ & $110.1(3)$ & $\mathrm{C} 8$ & $\mathrm{C} 7$ & C6 & $120.2(3)$ \\
\hline C38 & N6 & B2 & $126.9(3)$ & $\mathrm{C} 30$ & C31 & C38 & $120.1(3)$ \\
\hline C41 & N6 & C38 & $107.3(3)$ & $\mathrm{C} 30$ & C31 & C32 & $119.5(3)$ \\
\hline C41 & N6 & B2 & $125.8(3)$ & C38 & C31 & C32 & $120.4(3)$ \\
\hline N1 & $\mathrm{C} 4$ & $\mathrm{C} 5$ & $119.8(3)$ & N5 & $\mathrm{C} 27$ & $\mathrm{C} 26$ & $128.1(3)$ \\
\hline N1 & $\mathrm{C} 4$ & C3 & $108.4(3)$ & N5 & $\mathrm{C} 27$ & $\mathrm{C} 28$ & $108.5(3)$ \\
\hline C3 & $\mathrm{C} 4$ & C5 & $131.7(3)$ & $\mathrm{C} 26$ & $\mathrm{C} 27$ & $\mathrm{C} 28$ & $123.4(3)$ \\
\hline C14 & C13 & C12 & $108.3(3)$ & $\mathrm{C} 10$ & C9 & $\mathrm{C} 8$ & $120.2(3)$ \\
\hline N2 & C15 & C14 & $108.8(3)$ & C16 & C17 & $\mathrm{C} 18$ & $108.1(3)$ \\
\hline $\mathbf{N} 2$ & C15 & $\mathrm{C} 16$ & $128.1(3)$ & C9 & $\mathrm{C} 8$ & C7 & $120.0(3)$ \\
\hline C16 & $\mathrm{C} 15$ & C14 & $123.1(3)$ & $\mathrm{C} 23$ & $\mathrm{C} 24$ & $\mathrm{C} 25$ & $107.5(3)$ \\
\hline $\mathrm{C23}$ & $\mathrm{C} 20$ & C19 & $107.8(3)$ & C33 & $\mathrm{C} 32$ & C31 & $120.1(3)$ \\
\hline $\mathrm{C} 23$ & $\mathrm{C} 20$ & $\mathrm{C} 21$ & $110.4(3)$ & C37 & C32 & C33 & $118.5(3)$ \\
\hline C23 & $\mathrm{C} 20$ & $\mathrm{C} 22$ & $109.5(3)$ & C37 & C32 & C31 & $121.4(3)$ \\
\hline C19 & $\mathrm{C} 20$ & $\mathrm{C} 21$ & $109.7(3)$ & C26 & $\mathrm{C} 25$ & $\mathrm{C} 24$ & $108.4(3)$ \\
\hline C19 & $\mathrm{C} 20$ & $\mathrm{C} 22$ & $110.0(3)$ & $\mathrm{C} 35$ & C34 & C33 & $120.7(4)$ \\
\hline C21 & $\mathrm{C} 20$ & $\mathrm{C} 22$ & $109.5(3)$ & $\mathrm{C} 36$ & C37 & C32 & $120.5(4)$ \\
\hline C7 & C6 & $\mathrm{C} 5$ & $120.8(3)$ & N6 & C41 & $\mathrm{C} 40$ & $110.2(3)$ \\
\hline
\end{tabular}




\begin{tabular}{|c|c|c|c|c|c|c|c|}
\hline C11 & C6 & $\mathrm{C} 5$ & $120.1(3)$ & $\mathrm{C} 28$ & $\mathrm{C} 29$ & $\mathrm{C} 30$ & $108.8(3)$ \\
\hline C11 & C6 & $\mathrm{C} 7$ & $119.0(3)$ & C19 & C18 & $\mathrm{C} 17$ & $107.2(3)$ \\
\hline N4 & $\mathrm{C} 23$ & $\mathrm{C} 20$ & $120.1(3)$ & C35 & C36 & C37 & $120.1(4)$ \\
\hline N4 & $\mathrm{C} 23$ & $\mathrm{C} 24$ & $107.7(3)$ & C6 & $\mathrm{C} 11$ & $\mathrm{C} 10$ & $120.6(3)$ \\
\hline $\mathrm{C24}$ & $\mathrm{C} 23$ & $\mathrm{C} 20$ & $132.1(3)$ & $\mathrm{C} 29$ & $\mathrm{C} 28$ & $\mathrm{C} 27$ & $107.3(3)$ \\
\hline N5 & $\mathrm{C} 30$ & $\mathrm{C} 29$ & $107.1(3)$ & $\mathrm{F} 2$ & B1 & $\mathrm{N} 2$ & $109.2(3)$ \\
\hline C31 & $\mathrm{C} 30$ & N5 & $122.9(3)$ & $\mathrm{F} 2$ & B1 & N1 & $111.0(3)$ \\
\hline C31 & C30 & $\mathrm{C} 29$ & $129.8(3)$ & F1 & B1 & $\mathrm{F} 2$ & $107.5(3)$ \\
\hline N3 & C19 & $\mathrm{C} 20$ & $120.8(3)$ & F1 & B1 & $\mathrm{N} 2$ & $110.8(3)$ \\
\hline N3 & C19 & $\mathrm{C} 18$ & $107.7(3)$ & $\mathrm{F} 1$ & B1 & $\mathrm{N} 1$ & $110.6(3)$ \\
\hline C18 & C19 & $\mathrm{C} 20$ & $131.5(3)$ & N1 & B1 & $\mathrm{N} 2$ & $107.8(3)$ \\
\hline C13 & C14 & $\mathrm{C} 15$ & $107.9(3)$ & C34 & C35 & C36 & $120.0(4)$ \\
\hline $\mathrm{C4}$ & $\mathrm{C} 5$ & C6 & $120.5(3)$ & $\mathrm{Cl1}$ & $\mathrm{C} 42$ & $\mathrm{Cl} 2$ & $110.6(2)$ \\
\hline C12 & $\mathrm{C} 5$ & $\mathrm{C} 4$ & $120.4(3)$ & $\mathrm{Cl1}$ & $\mathrm{C} 42$ & $\mathrm{Cl} 3$ & $111.0(2)$ \\
\hline $\mathrm{C12}$ & $\mathrm{C} 5$ & C6 & $119.1(3)$ & $\mathrm{Cl} 3$ & $\mathrm{C} 42$ & $\mathrm{Cl} 2$ & $110.12(19)$ \\
\hline $\mathbf{N 3}$ & $\mathrm{C} 16$ & $\mathrm{C} 15$ & $127.2(3)$ & C9 & $\mathrm{C} 10$ & $\mathrm{C} 11$ & $119.9(3)$ \\
\hline N3 & C16 & C17 & $106.4(3)$ & F3 & B2 & N5 & $109.8(3)$ \\
\hline C17 & C16 & $\mathrm{C} 15$ & $126.5(3)$ & F3 & B2 & N6 & $110.7(3)$ \\
\hline $\mathbf{N} 2$ & C12 & $\mathrm{C} 13$ & $107.4(3)$ & $\mathrm{F} 4$ & $\mathrm{~B} 2$ & F3 & $108.0(3)$ \\
\hline C5 & C12 & $\mathrm{N} 2$ & $122.7(3)$ & $\mathrm{F} 4$ & B2 & N5 & $110.1(3)$ \\
\hline C5 & C12 & $\mathrm{C} 13$ & $129.8(3)$ & $\mathrm{F} 4$ & B2 & N6 & $110.9(3)$ \\
\hline $\mathrm{C2}$ & $\mathrm{C} 3$ & $\mathrm{C} 4$ & $107.3(3)$ & N6 & B2 & N5 & $107.3(3)$ \\
\hline N1 & $\mathrm{C} 1$ & $\mathrm{C} 2$ & $109.9(3)$ & & & & \\
\hline
\end{tabular}


Table S4: Bond Lengths for 1-Cu

\begin{tabular}{cccccc}
\hline Atom & Atom & Length/A & Atom & Atom & Length/A \\
\hline C1 & N1 & $1.338(5)$ & C45 & N7 & $1.396(5)$ \\
\hline C1 & C2 & $1.402(6)$ & C45 & C46 & $1.407(6)$ \\
\hline C2 & C3 & $1.354(6)$ & C46 & C53 & $1.391(6)$ \\
\hline C3 & C4 & $1.410(6)$ & C46 & C47 & $1.487(6)$ \\
\hline C4 & N1 & $1.399(5)$ & C47 & C48 & $1.390(6)$ \\
\hline C4 & C5 & $1.404(6)$ & C47 & C52 & $1.393(6)$ \\
\hline C5 & C12 & $1.389(6)$ & C48 & C49 & $1.377(7)$ \\
\hline C5 & C6 & $1.501(5)$ & C49 & C50 & $1.358(8)$ \\
\hline C6 & C7 & $1.383(6)$ & C50 & C51 & $1.375(9)$ \\
\hline C6 & C11 & $1.385(6)$ & C51 & C52 & $1.394(7)$ \\
\hline C7 & C8 & $1.401(6)$ & C53 & N8 & $1.406(5)$ \\
\hline C8 & C9 & $1.353(7)$ & C53 & C54 & $1.436(6)$ \\
\hline C9 & C10 & $1.350(7)$ & C54 & C55 & $1.353(6)$ \\
\hline C10 & C11 & $1.390(6)$ & C55 & C56 & $1.425(6)$ \\
\hline C12 & N2 & $1.402(5)$ & C56 & N8 & $1.350(5)$ \\
\hline C12 & C13 & $1.431(6)$ & C56 & C57 & $1.425(6)$ \\
\hline C13 & C14 & $1.358(6)$ & C57 & N9 & $1.379(5)$ \\
\hline C14 & C15 & $1.419(5)$ & C57 & C58 & $1.396(6)$ \\
\hline C15 & N2 & $1.356(5)$ & C58 & C59 & $1.378(6)$ \\
\hline C15 & C16 & $1.443(6)$ & C59 & C60 & $1.379(6)$ \\
\hline C16 & N3 & $1.371(5)$ & C60 & N9 & $1.361(5)$ \\
\hline C16 & C17 & $1.376(6)$ & C60 & C61 & $1.494(6)$ \\
\hline C17 & C18 & $1.398(6)$ & C61 & C64 & $1.521(6)$ \\
\hline C18 & C19 & $1.361(6)$ & C61 & C63 & $1.531(6)$ \\
\hline C19 & N3 & $1.364(5)$ & C61 & C62 & $1.542(6)$ \\
\hline C19 & C20 & $1.505(6)$ & C64 & N10 & $1.364(5)$ \\
\hline C20 & C23 & $1.505(6)$ & C64 & C65 & $1.369(6)$ \\
\hline C20 & C22 & $1.529(6)$ & C65 & C66 & $1.386(6)$ \\
\hline C20 & C21 & $1.546(6)$ & C66 & C67 & $1.375(6)$ \\
\hline C23 & C24 & $1.363(6)$ & C67 & N10 & $1.370(5)$ \\
\hline C23 & N4 & $1.369(5)$ & C67 & C68 & $1.441(5)$ \\
\hline & & & & &
\end{tabular}




\begin{tabular}{|c|c|c|c|c|c|}
\hline $\mathrm{C24}$ & $\mathrm{C} 25$ & $1.387(6)$ & C68 & N11 & $1.348(5)$ \\
\hline $\mathrm{C25}$ & C26 & $1.375(6)$ & C68 & C69 & $1.424(5)$ \\
\hline $\mathrm{C} 26$ & N4 & $1.373(5)$ & C69 & C70 & $1.358(6)$ \\
\hline $\mathrm{C} 26$ & $\mathrm{C} 27$ & $1.438(6)$ & $\mathrm{C} 70$ & C71 & $1.430(6)$ \\
\hline C27 & N5 & $1.358(5)$ & C71 & $\mathrm{C} 72$ & $1.382(5)$ \\
\hline C27 & C28 & $1.415(6)$ & C71 & N11 & $1.422(5)$ \\
\hline $\mathrm{C} 28$ & C29 & $1.351(6)$ & $\mathrm{C} 72$ & C79 & $1.403(5)$ \\
\hline $\mathrm{C29}$ & C30 & $1.424(6)$ & $\mathrm{C} 72$ & C73 & $1.506(5)$ \\
\hline C30 & C31 & $1.395(6)$ & C73 & C74 & $1.384(5)$ \\
\hline C30 & N5 & $1.407(5)$ & C73 & C78 & $1.389(6)$ \\
\hline C31 & C38 & $1.409(6)$ & $\mathrm{C} 74$ & $\mathrm{C} 75$ & $1.383(5)$ \\
\hline C31 & C32 & $1.494(6)$ & $\mathrm{C} 75$ & C76 & $1.380(6)$ \\
\hline C32 & C37 & $1.375(6)$ & C76 & C77 & $1.381(6)$ \\
\hline C32 & C33 & $1.380(6)$ & C77 & C78 & $1.382(6)$ \\
\hline C33 & C34 & $1.384(6)$ & C79 & N12 & $1.393(4)$ \\
\hline C34 & C35 & $1.353(7)$ & C79 & $\mathrm{C} 80$ & $1.405(5)$ \\
\hline C35 & C36 & $1.363(7)$ & $\mathrm{C} 80$ & $\mathrm{C} 81$ & $1.371(6)$ \\
\hline C36 & C37 & $1.384(6)$ & $\mathrm{C} 81$ & C82 & $1.381(6)$ \\
\hline C38 & N6 & $1.389(5)$ & $\mathrm{C} 82$ & N12 & $1.341(5)$ \\
\hline C38 & C39 & $1.413(6)$ & $\mathrm{Cu} 1$ & N12 & $1.916(3)$ \\
\hline C39 & $\mathrm{C} 40$ & $1.368(6)$ & $\mathrm{Cu} 1$ & N1 & $1.933(3)$ \\
\hline C40 & C41 & $1.400(6)$ & $\mathrm{Cu} 1$ & $\mathrm{~N} 2$ & $1.972(3)$ \\
\hline C41 & N6 & $1.345(5)$ & $\mathrm{Cu} 1$ & N11 & $1.975(3)$ \\
\hline $\mathrm{C42}$ & N7 & $1.358(5)$ & $\mathrm{Cu} 2$ & N7 & $1.930(3)$ \\
\hline $\mathrm{C42}$ & C43 & $1.387(6)$ & $\mathrm{Cu} 2$ & N6 & $1.933(3)$ \\
\hline C43 & C44 & $1.376(6)$ & $\mathrm{Cu} 2$ & N8 & $1.967(3)$ \\
\hline C44 & $\mathrm{C} 45$ & $1.405(5)$ & $\mathrm{Cu} 2$ & N5 & $1.983(3)$ \\
\hline
\end{tabular}


Table S5: Bond Angles for 1-Cu

\begin{tabular}{|c|c|c|c|c|c|c|c|}
\hline Atom & Atom & Atom & Angle ${ }^{\circ}$ & Atom & Atom & Atom & Angle $/^{\circ}$ \\
\hline N1 & $\mathrm{C} 1$ & $\mathrm{C} 2$ & $110.8(4)$ & C55 & C54 & C53 & $107.9(4)$ \\
\hline C3 & $\mathrm{C} 2$ & $\mathrm{C} 1$ & $107.1(4)$ & C54 & C55 & C56 & $107.0(4)$ \\
\hline $\mathrm{C} 2$ & $\mathrm{C} 3$ & $\mathrm{C} 4$ & $107.5(4)$ & N8 & C56 & C55 & $110.5(4)$ \\
\hline N1 & $\mathrm{C} 4$ & C5 & $123.3(4)$ & N8 & C56 & C57 & $124.9(4)$ \\
\hline N1 & $\mathrm{C} 4$ & C3 & $108.3(4)$ & C55 & C56 & C57 & $124.6(4)$ \\
\hline C5 & $\mathrm{C} 4$ & C3 & $128.4(4)$ & N9 & C57 & C58 & $105.1(4)$ \\
\hline $\mathrm{C12}$ & C5 & $\mathrm{C} 4$ & 125.6() & N9 & $\mathrm{C} 57$ & C56 & $126.8(4)$ \\
\hline $\mathrm{C12}$ & C5 & C6 & $117.3(4)$ & C58 & C57 & C56 & $127.9(4)$ \\
\hline C4 & $\mathrm{C} 5$ & C6 & $117.0(4)$ & C59 & C58 & C57 & $108.3(4)$ \\
\hline C7 & C6 & $\mathrm{C} 11$ & $118.4(4)$ & C58 & C59 & C60 & $109.1(4)$ \\
\hline C7 & C6 & C5 & $120.3(4)$ & N9 & C60 & C59 & $105.9(4)$ \\
\hline C11 & C6 & $\mathrm{C} 5$ & $121.1(4)$ & N9 & C60 & C61 & $122.2(4)$ \\
\hline C6 & C7 & C8 & $120.1(5)$ & C59 & C60 & C61 & $131.6(4)$ \\
\hline C9 & $\mathrm{C} 8$ & $\mathrm{C} 7$ & $119.8(5)$ & C60 & C61 & C64 & $112.3(4)$ \\
\hline C10 & C9 & $\mathrm{C} 8$ & $121.0(5)$ & C60 & C61 & C63 & $109.6(4)$ \\
\hline C9 & $\mathrm{C} 10$ & $\mathrm{C} 11$ & $120.3(5)$ & C64 & C61 & C63 & $109.6(4)$ \\
\hline C6 & C11 & C10 & $120.2(5)$ & C60 & C61 & C62 & $109.3(4)$ \\
\hline C5 & $\mathrm{C} 12$ & N2 & $124.1(4)$ & C64 & C61 & C62 & $107.2(4)$ \\
\hline $\mathrm{C5}$ & $\mathrm{C} 12$ & C13 & $127.4(4)$ & C63 & C61 & C62 & $108.8(4)$ \\
\hline N2 & C12 & C13 & $108.3(4)$ & N10 & C64 & C65 & $106.7(4)$ \\
\hline C14 & C13 & C12 & $108.0(4)$ & N10 & C64 & C61 & $121.5(4)$ \\
\hline $\mathrm{C13}$ & $\mathrm{C} 14$ & $\mathrm{C} 15$ & $106.5(4)$ & C65 & C64 & C61 & $131.2(4)$ \\
\hline $\mathbf{N 2}$ & C15 & C14 & $111.1(4)$ & C64 & C65 & C66 & $108.5(4)$ \\
\hline N2 & $\mathrm{C} 15$ & C16 & $124.2(4)$ & C67 & C66 & C65 & $108.0(4)$ \\
\hline C14 & $\mathrm{C} 15$ & C16 & $124.6(4)$ & N10 & C67 & C66 & $106.5(4)$ \\
\hline N3 & C16 & $\mathrm{C} 17$ & $106.8(4)$ & N10 & C67 & C68 & $125.4(4)$ \\
\hline N3 & C16 & $\mathrm{C} 15$ & $125.6(4)$ & C66 & C67 & C68 & $127.9(4)$ \\
\hline C17 & C16 & $\mathrm{C} 15$ & $127.5(4)$ & N11 & C68 & C69 & $110.9(3)$ \\
\hline C16 & $\mathrm{C} 17$ & C18 & $107.2(4)$ & N11 & C68 & C67 & $125.1(4)$ \\
\hline
\end{tabular}




\begin{tabular}{|c|c|c|c|c|c|c|c|}
\hline C19 & C18 & $\mathrm{C} 17$ & $108.8(4)$ & C69 & C68 & C67 & $124.0(4)$ \\
\hline C18 & C19 & $\mathrm{N} 3$ & $106.8(4)$ & $\mathrm{C} 70$ & C69 & C68 & $107.2(4)$ \\
\hline C18 & C19 & $\mathrm{C} 20$ & $132.3(4)$ & C69 & $\mathrm{C} 70$ & C71 & $107.6(4)$ \\
\hline N3 & C19 & $\mathrm{C} 20$ & $120.9(4)$ & $\mathrm{C} 72$ & C71 & N11 & $124.4(4)$ \\
\hline C19 & $\mathrm{C} 20$ & $\mathrm{C} 23$ & $109.6(3)$ & $\mathrm{C} 72$ & C71 & $\mathrm{C} 70$ & $127.5(4)$ \\
\hline C19 & $\mathrm{C} 20$ & $\mathrm{C} 22$ & $109.6(4)$ & N11 & C71 & $\mathrm{C} 70$ & $108.1(4)$ \\
\hline $\mathrm{C23}$ & C20 & $\mathrm{C} 22$ & $109.9(4)$ & C71 & C72 & C79 & $125.6(4)$ \\
\hline C19 & $\mathrm{C} 20$ & $\mathrm{C} 21$ & $108.7(4)$ & C71 & $\mathrm{C} 72$ & $\mathrm{C} 73$ & $118.1(4)$ \\
\hline $\mathrm{C23}$ & $\mathrm{C} 20$ & $\mathrm{C} 21$ & $110.2(4)$ & C79 & $\mathrm{C} 72$ & C73 & $116.2(4)$ \\
\hline $\mathrm{C22}$ & $\mathrm{C} 20$ & $\mathrm{C} 21$ & $108.8(4)$ & C74 & C73 & C78 & $119.2(4)$ \\
\hline $\mathrm{C24}$ & $\mathrm{C} 23$ & $\mathrm{~N} 4$ & $107.2(4)$ & C74 & C73 & $\mathrm{C} 72$ & $121.7(4)$ \\
\hline $\mathrm{C24}$ & $\mathrm{C} 23$ & $\mathrm{C} 20$ & $132.6(4)$ & C78 & C73 & $\mathrm{C} 72$ & $119.1(4)$ \\
\hline N4 & $\mathrm{C} 23$ & $\mathrm{C} 20$ & $120.2(4)$ & C73 & C74 & $\mathrm{C} 75$ & $120.4(4)$ \\
\hline $\mathrm{C23}$ & $\mathrm{C} 24$ & $\mathrm{C} 25$ & $108.0(4)$ & C76 & $\mathrm{C} 75$ & C74 & $120.2(4)$ \\
\hline C26 & $\mathrm{C} 25$ & $\mathrm{C} 24$ & $108.6(4)$ & $\mathrm{C} 75$ & C76 & C77 & $119.6(4)$ \\
\hline N4 & $\mathrm{C} 26$ & $\mathrm{C} 25$ & $106.2(4)$ & C76 & C77 & $\mathrm{C} 78$ & $120.4(4)$ \\
\hline N4 & $\mathrm{C} 26$ & $\mathrm{C} 27$ & $126.6(4)$ & C77 & $\mathrm{C} 78$ & $\mathrm{C} 73$ & $120.2(4)$ \\
\hline $\mathrm{C25}$ & $\mathrm{C} 26$ & $\mathrm{C} 27$ & $127.0(4)$ & $\mathrm{N} 12$ & C79 & $\mathrm{C} 72$ & $123.1(4)$ \\
\hline N5 & $\mathrm{C} 27$ & $\mathrm{C} 28$ & $110.5(4)$ & N12 & C79 & $\mathrm{C} 80$ & $108.7(3)$ \\
\hline N5 & $\mathrm{C} 27$ & $\mathrm{C} 26$ & $125.7(4)$ & $\mathrm{C} 72$ & C79 & $\mathrm{C} 80$ & $128.1(4)$ \\
\hline $\mathrm{C} 28$ & C27 & $\mathrm{C} 26$ & $123.8(4)$ & C81 & C80 & C79 & $107.0(4)$ \\
\hline $\mathrm{C29}$ & $\mathrm{C} 28$ & $\mathrm{C} 27$ & $107.6(4)$ & $\mathrm{C} 80$ & $\mathrm{C} 81$ & $\mathrm{C} 82$ & $106.8(4)$ \\
\hline $\mathrm{C} 28$ & $\mathrm{C} 29$ & $\mathrm{C} 30$ & $107.4(4)$ & $\mathrm{N} 12$ & $\mathrm{C} 82$ & $\mathrm{C} 81$ & $111.7(4)$ \\
\hline C31 & $\mathrm{C} 30$ & N5 & $124.9(4)$ & $\mathrm{N} 12$ & $\mathrm{Cu} 1$ & N1 & $145.79(14)$ \\
\hline C31 & $\mathrm{C} 30$ & $\mathrm{C} 29$ & $126.1(4)$ & $\mathrm{N} 12$ & $\mathrm{Cu} 1$ & $\mathrm{~N} 2$ & $97.55(13)$ \\
\hline N5 & $\mathrm{C} 30$ & $\mathrm{C} 29$ & $108.7(4)$ & N1 & $\mathrm{Cu} 1$ & $\mathrm{~N} 2$ & $91.91(14)$ \\
\hline C30 & C31 & $\mathrm{C} 38$ & $125.7(4)$ & $\mathrm{N} 12$ & $\mathrm{Cu} 1$ & N11 & $92.29(13)$ \\
\hline C30 & C31 & $\mathrm{C} 32$ & $117.4(4)$ & N1 & $\mathrm{Cu} 1$ & N11 & $100.17(14)$ \\
\hline C38 & $\mathrm{C} 31$ & $\mathrm{C} 32$ & $116.9(4)$ & $\mathrm{N} 2$ & $\mathrm{Cu} 1$ & N11 & $142.10(13)$ \\
\hline C37 & C32 & $\mathrm{C} 33$ & $117.3(4)$ & N7 & $\mathrm{Cu} 2$ & N6 & $143.13(15)$ \\
\hline C37 & $\mathrm{C} 32$ & $\mathrm{C} 31$ & $121.1(4)$ & N7 & $\mathrm{Cu} 2$ & N8 & $91.37(14)$ \\
\hline C33 & $\mathrm{C} 32$ & $\mathrm{C} 31$ & $121.6(4)$ & N6 & $\mathrm{Cu} 2$ & N8 & $97.41(14)$ \\
\hline C32 & $\mathrm{C} 33$ & $\mathrm{C} 34$ & $120.7(5)$ & N7 & $\mathrm{Cu} 2$ & N5 & $102.71(14)$ \\
\hline C35 & C34 & $\mathrm{C} 33$ & $120.9(5)$ & N6 & $\mathrm{Cu} 2$ & N5 & $92.71(14)$ \\
\hline
\end{tabular}




\begin{tabular}{|c|c|c|c|c|c|c|c|}
\hline C34 & $\mathrm{C} 35$ & $\mathrm{C} 36$ & $119.5(5)$ & N8 & $\mathrm{Cu} 2$ & N5 & $140.93(14)$ \\
\hline C35 & C36 & C37 & $119.8(5)$ & C1 & N1 & $\mathrm{C} 4$ & $106.3(3)$ \\
\hline C32 & C37 & C36 & $121.7(5)$ & $\mathrm{C} 1$ & N1 & $\mathrm{Cu} 1$ & $127.8(3)$ \\
\hline N6 & C38 & C31 & $123.4(4)$ & $\mathrm{C} 4$ & N1 & $\mathrm{Cu} 1$ & $123.6(3)$ \\
\hline N6 & C38 & C39 & $108.6(4)$ & C15 & $\mathrm{N} 2$ & C12 & $106.1(3)$ \\
\hline C31 & C38 & C39 & $128.1(4)$ & C15 & $\mathrm{N} 2$ & $\mathrm{Cu} 1$ & $129.3(3)$ \\
\hline C40 & C39 & C38 & $107.1(4)$ & C12 & $\mathrm{N} 2$ & $\mathrm{Cu} 1$ & $120.4(3)$ \\
\hline C39 & $\mathrm{C} 40$ & $\mathrm{C} 41$ & $107.0(4)$ & C19 & N3 & C16 & $110.4(4)$ \\
\hline N6 & C41 & $\mathrm{C} 40$ & $110.7(4)$ & $\mathrm{C} 23$ & N4 & $\mathrm{C} 26$ & $110.0(4)$ \\
\hline N7 & $\mathrm{C} 42$ & $\mathrm{C} 43$ & $110.7(4)$ & C27 & N5 & $\mathrm{C} 30$ & $105.8(3)$ \\
\hline C44 & $\mathrm{C} 43$ & $\mathrm{C} 42$ & $107.3(4)$ & C27 & N5 & $\mathrm{Cu} 2$ & $131.7(3)$ \\
\hline C43 & $\mathrm{C} 44$ & $\mathrm{C} 45$ & $107.0(4)$ & C30 & N5 & $\mathrm{Cu} 2$ & $122.1(3)$ \\
\hline N7 & $\mathrm{C} 45$ & $\mathrm{C} 44$ & $108.8(4)$ & C41 & N6 & C38 & $106.6(3)$ \\
\hline N7 & $\mathrm{C} 45$ & $\mathrm{C} 46$ & $123.7(4)$ & C41 & N6 & $\mathrm{Cu} 2$ & $126.0(3)$ \\
\hline C44 & $\mathrm{C} 45$ & $\mathrm{C} 46$ & $127.4(4)$ & C38 & N6 & $\mathrm{Cu} 2$ & $124.8(3)$ \\
\hline C53 & $\mathrm{C} 46$ & $\mathrm{C} 45$ & $124.7(4)$ & C42 & N7 & $\mathrm{C} 45$ & $106.1(3)$ \\
\hline C53 & $\mathrm{C} 46$ & $\mathrm{C} 47$ & $116.8(4)$ & $\mathrm{C} 42$ & N7 & $\mathrm{Cu} 2$ & $128.2(3)$ \\
\hline C45 & $\mathrm{C} 46$ & $\mathrm{C} 47$ & $118.5(4)$ & $\mathrm{C} 45$ & N7 & $\mathrm{Cu} 2$ & $123.5(3)$ \\
\hline C48 & $\mathrm{C} 47$ & C52 & $118.8(5)$ & C56 & N8 & C53 & $106.8(4)$ \\
\hline C48 & $\mathrm{C} 47$ & $\mathrm{C} 46$ & $119.9(4)$ & C56 & N8 & $\mathrm{Cu} 2$ & $127.9(3)$ \\
\hline C52 & $\mathrm{C} 47$ & $\mathrm{C} 46$ & $121.3(4)$ & C53 & N8 & $\mathrm{Cu} 2$ & $120.5(3)$ \\
\hline C49 & $\mathrm{C} 48$ & $\mathrm{C} 47$ & $120.3(5)$ & C60 & N9 & $\mathrm{C} 57$ & $111.6(4)$ \\
\hline C50 & C49 & C48 & $121.0(6)$ & C64 & N10 & C67 & $110.4(4)$ \\
\hline C49 & $\mathrm{C} 50$ & C51 & $119.6(6)$ & C68 & N11 & C71 & $106.0(3)$ \\
\hline C50 & C51 & C52 & $120.6(6)$ & C68 & N11 & $\mathrm{Cu} 1$ & $130.5(3)$ \\
\hline C47 & C52 & C51 & $119.4(5)$ & C71 & N11 & $\mathrm{Cu} 1$ & $120.9(3)$ \\
\hline C46 & C53 & N8 & $124.2(4)$ & C82 & N12 & C79 & $105.8(3)$ \\
\hline C46 & C53 & C54 & $128.1(4)$ & C82 & N12 & $\mathrm{Cu} 1$ & $128.0(3)$ \\
\hline N8 & C53 & C54 & $107.6(4)$ & C79 & N12 & $\mathrm{Cu} 1$ & $125.6(3)$ \\
\hline
\end{tabular}




\section{ESR Spectra of compound 1-Cu}

Further the ESR spectra of 1-Cu was recorded in Bruker EMX Plus series equipped with temperature controller. Figure S9 shows the ESR spectra for the 1-Cu in the toluene solution recorded at $100 \mathrm{~K}$. The complex 1-Cu exhibits the typical ESR spectrum due to the Cu $\mathrm{Cu}^{\mathrm{II}} \mathrm{metal}$ atom with $S=1 / 2$ state. The presence of two non-interacting copper atoms in slightly different coordination environment is responsible for further resonances in the ESR spectra.

The ESR spectra of 1-Cu can be simulated by spin Hamiltonian as the molecule contains copper metal atoms in +2 oxidation state $(\mathrm{S}=1 / 2$ spin state) and it can be written as,

$$
\mathrm{H}_{\mathrm{S}}=\mu_{\mathrm{B}} \mathrm{S} * \mathrm{~B}_{0} * \mathrm{~g}+\mathrm{S} * \mathrm{~A}^{\mathrm{Cu}} * \mathrm{I}+\mathrm{H}_{\mathrm{N}}
$$

where $\mu_{\mathrm{B}}$, is Bohr magneton, $\mathrm{S}$ is the electron spin operator, $\mathrm{B}_{0}$ is applied magnetic field, $\mathrm{g}$ is the $\mathrm{g}$ tensor of electron spin, $\mathrm{A}$ is for hyperfine coupling tensor, and the $\mathrm{I}$ is for $\mathrm{Cu}^{\mathrm{II}}$ nuclear spin operators. For simplifying the calculations, the copper quadruple interaction is neglected. No distinction is made between two isotopes of copper ${ }^{63} \mathrm{Cu}$ and ${ }^{65} \mathrm{Cu}$, as their individual contributions were not observed in the experimental spectra. The third term $\mathrm{H}_{\mathrm{N}}$ is also negligible as it describes the ${ }^{13} \mathrm{C}$ and ${ }^{1} \mathrm{H}$ interactions in the system. The $\mathrm{g}$ and $\mathrm{A}$ tensors were considered to be co-axial. The experimental data is well explainable after these assumptions are made. The ESR spectra of 1-Cu is simulated considering two independent copper metal nuclei with equal weightage. The parameters used for simulating the spectrum for one copper, $\mathrm{S}=1 / 2, \mathrm{~g}=(2.01,2.075,2.242), \mathrm{A}=$ $(5,5,210) \mathrm{MHz}$ and line width $=(2.18,2.18)$ and for other copper, $\mathrm{S}=1 / 2, \mathrm{~g}=(2.06,2.075,2.35), \mathrm{A}=(20,20,210) \mathrm{MHz}$ and line width $=(2.1$, 2.18) $\mathrm{mT}$. 


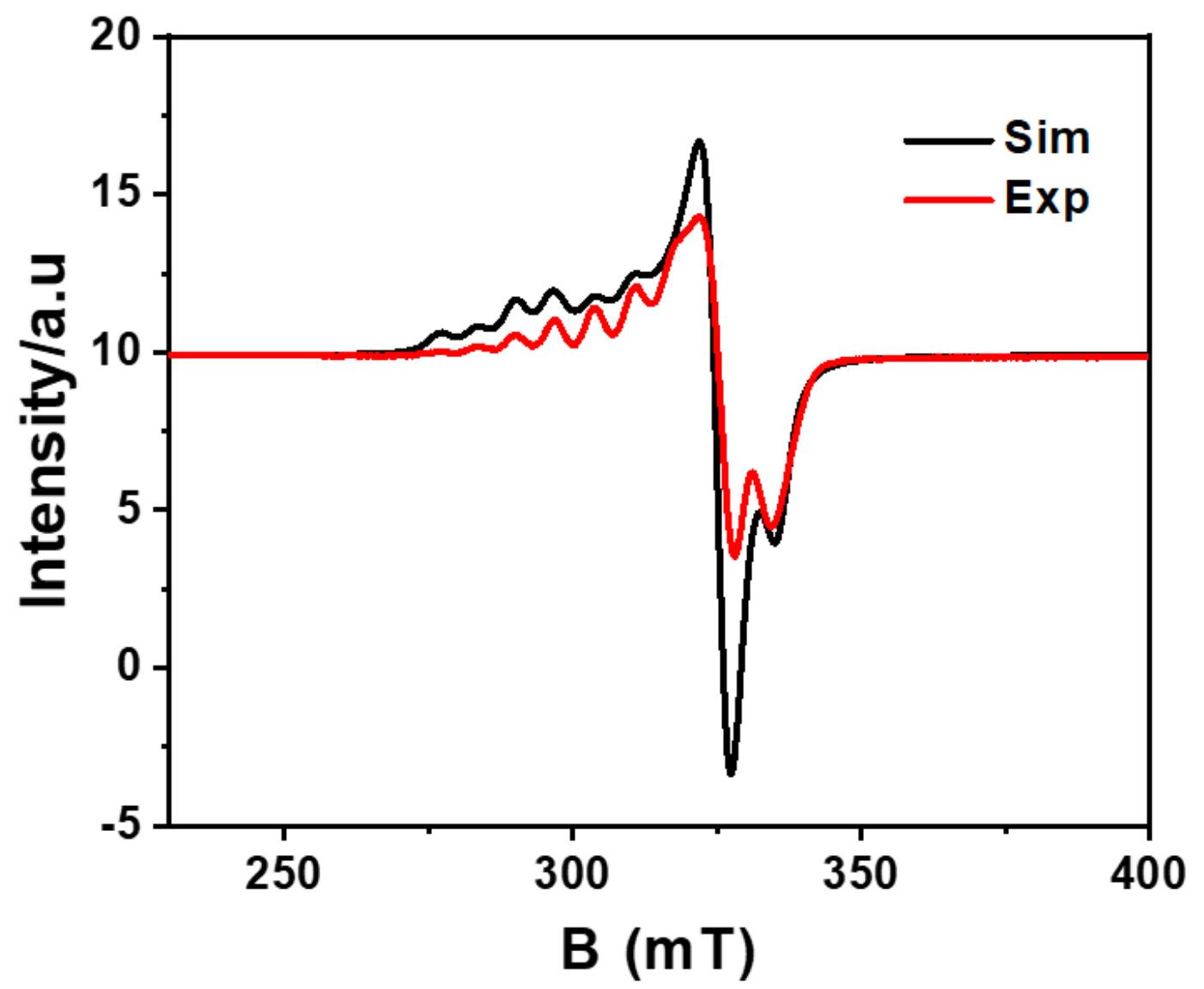

Figure S9: ESR spectra experimental (red) and simulated (black). 


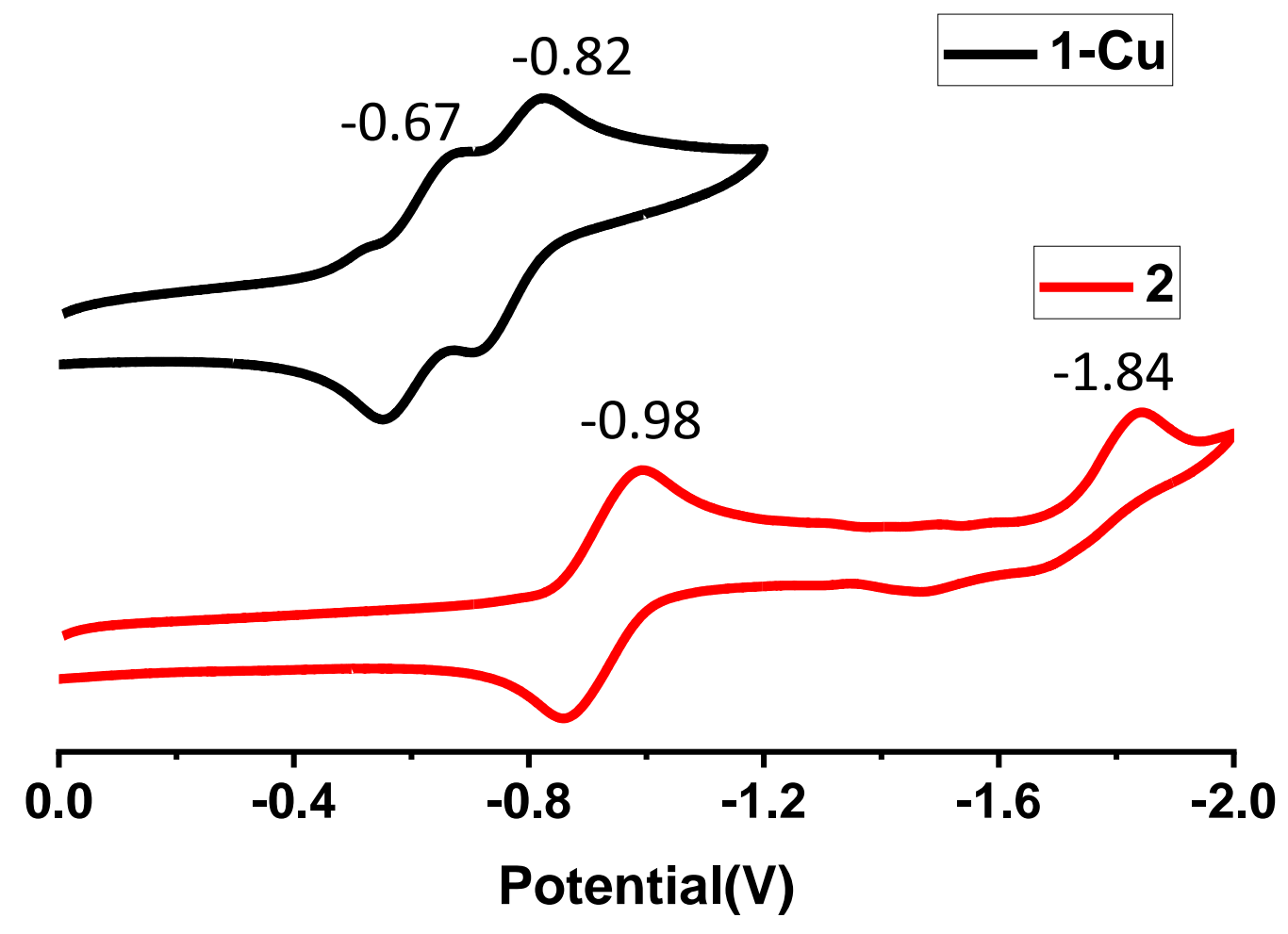

Figure S10: Comparison of cyclic voltammograms of compounds $\mathbf{2}$ and 1-Cu recorded in $\mathrm{CH}_{2} \mathrm{Cl}_{2}$ containing $0.1 \mathrm{M}$ TBAP as supporting electrolyte recorded at $50 \mathrm{mV} / \mathrm{s}$ scan speed. 


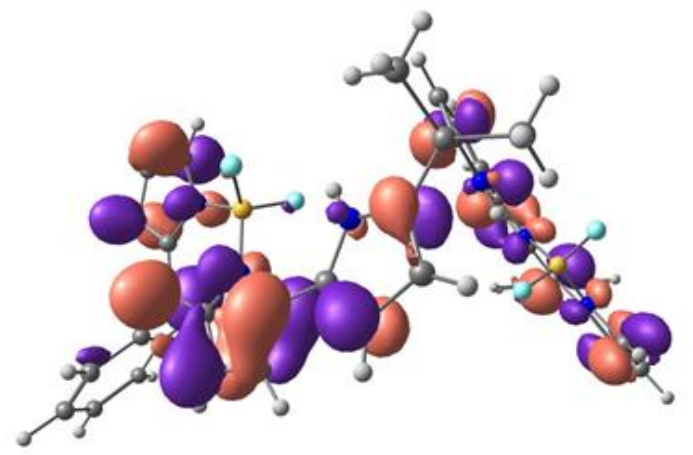

LUMO +1

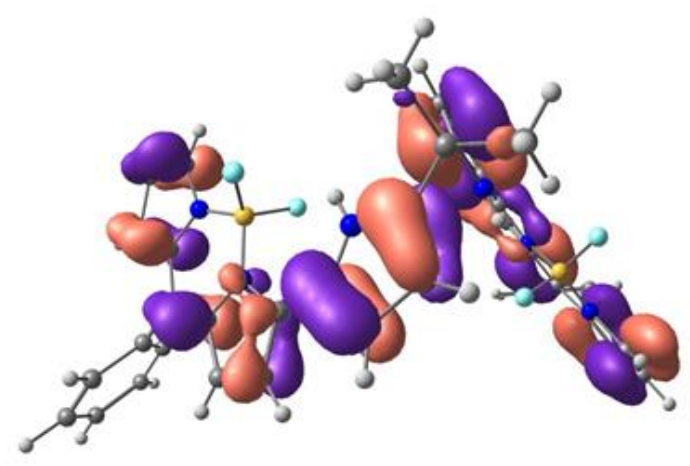

HOMO
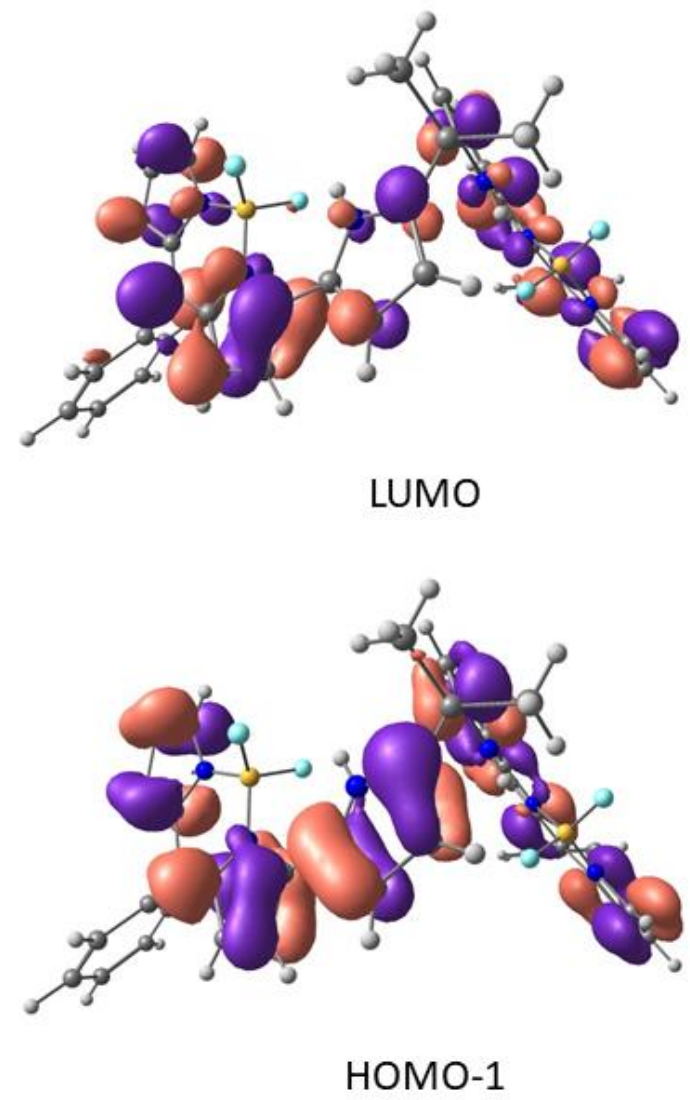

Figure S11: Frontier Molecular orbital diagram of compound 2. 


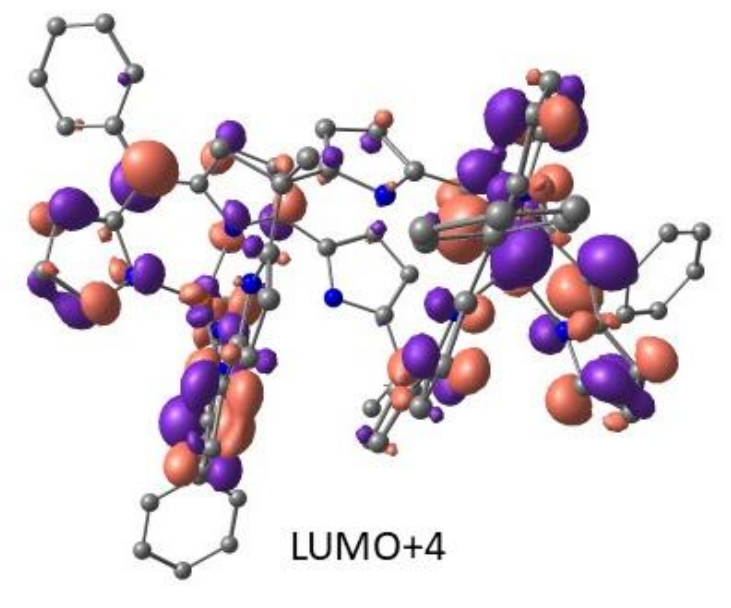

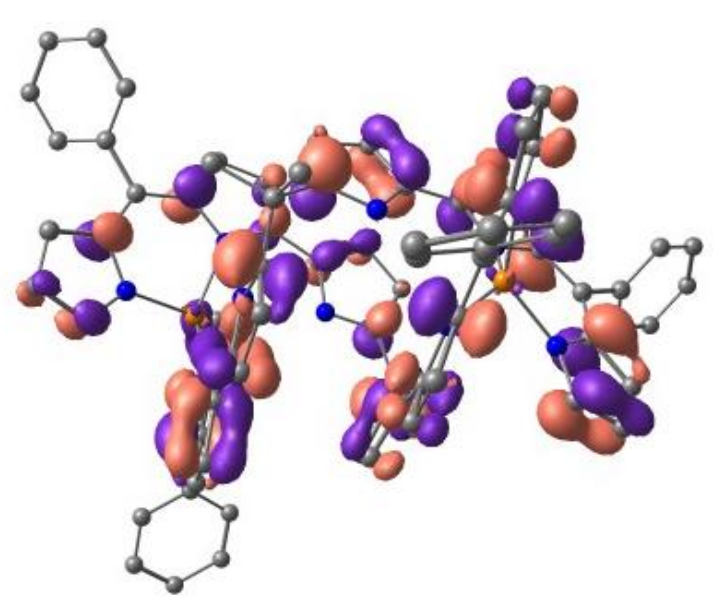

HOMO-2

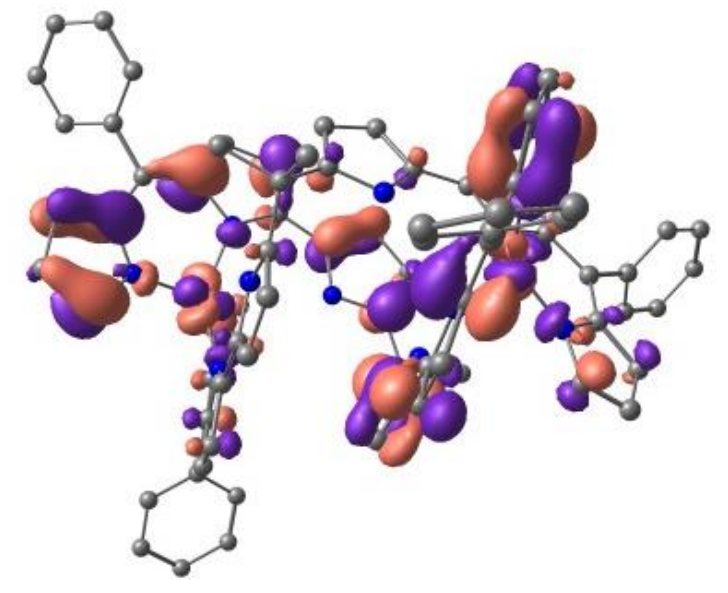

HOMO-3

Figure S12: HOMO-2, HOMO-3, and LUMO+4 of 1-Cu. 

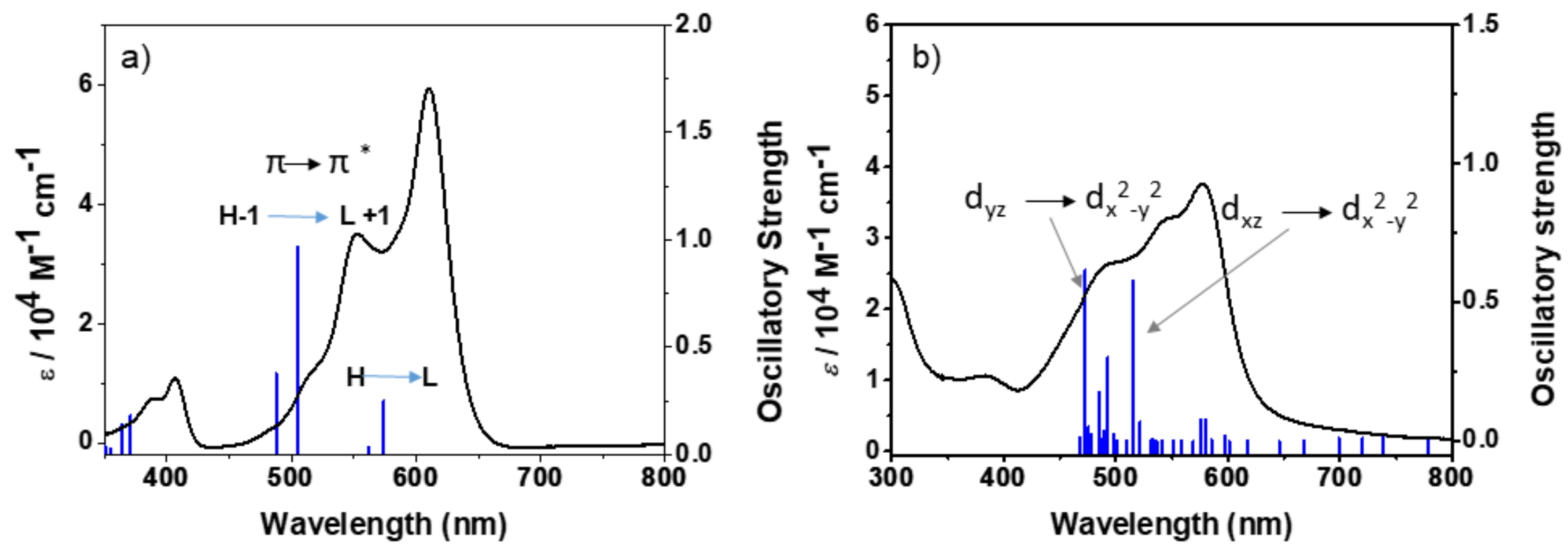

Figure S13: Calculated excitations (blue vertical lines, B3LYP/uB3LYP), experimental UV/vis absorption spectra (black line) and interpretation for compounds (a) 2 and (b) 1-Cu using ORCA 4.2 package. 
Table S6: TD-DFT transition data of Compound 2

\begin{tabular}{|c|c|c|c|}
\hline $\begin{array}{l}\text { Wavelength } \\
\quad(\mathrm{nm})\end{array}$ & $\begin{array}{l}\text { Osc. } \\
\text { Strength }\end{array}$ & Major contribs & Minor contribs \\
\hline 574.1338 & 0.2504 & H-1->L+1 (11\%), HOMO->LUMO (83\%) & HOMO->L+1 (6\%) \\
\hline 561.9553 & 0.0326 & H-1->LUMO (35\%), HOMO->L+1 (59\%) & HOMO->LUMO (5\%) \\
\hline 504.9244 & 0.9686 & H-1->L+1 (80\%), HOMO->LUMO (13\%) & H-1->LUMO (4\%), HOMO->L+1 (2\%) \\
\hline 487.7235 & 0.3776 & H-1->LUMO (60\%), HOMO->L+1 (33\%) & $\mathrm{H}-1->\mathrm{L}+1(8 \%)$ \\
\hline 370.8992 & 0.1875 & H-2->LUMO (67\%) & $\begin{array}{c}\mathrm{H}-4->\mathrm{LUMO}(8 \%), \mathrm{H}-4->\mathrm{L}+1(5 \%), \mathrm{H}-3->\mathrm{L}+1 \\
(5 \%), \mathrm{H}-2->\mathrm{L}+1(9 \%)\end{array}$ \\
\hline 364.3486 & 0.1425 & H-3->LUMO (14\%), H-2->L+1 (50\%) & $\begin{array}{c}\text { H-5->LUMO (8\%), H-5->L+1 (8\%), H-3->L+1 } \\
(4 \%), \mathrm{H}-2->\text { LUMO }(6 \%)\end{array}$ \\
\hline 354.8082 & 0.0298 & $\begin{array}{c}\text { H-3->LUMO }(31 \%), \mathrm{H}-3->\mathrm{L}+1(16 \%), \mathrm{H}-2->\mathrm{LUMO} \\
(11 \%), \mathrm{H}-2->\mathrm{L}+1(27 \%)\end{array}$ & H-5->LUMO (6\%), H-5->L+1 (6\%) \\
\hline 350.8325 & 0.0391 & $\begin{array}{l}\text { H-4->LUMO (23\%), H-4->L+1 (13\%), H-3->LUMO } \\
\text { (20\%), H-3->L+1 (21\%), H-2->LUMO (12\%) }\end{array}$ & $\mathrm{H}-2->\mathrm{L}+1(7 \%)$ \\
\hline 348.6031 & 0.0669 & $\begin{array}{c}\text { H-4->LUMO }(29 \%), \mathrm{H}-4->\mathrm{L}+1(17 \%), \mathrm{H}-3->\mathrm{LUMO} \\
(24 \%), \mathrm{H}-3->\mathrm{L}+1(17 \%)\end{array}$ & H-5->LUMO (4\%) \\
\hline 346.3246 & 0.0897 & $\begin{array}{c}\mathrm{H}-5->\mathrm{LUMO}(28 \%), \mathrm{H}-5->\mathrm{L}+1 \\
(26 \%)\end{array}$ & $\begin{aligned} \text { H-10->LUMO } & (3 \%), \mathrm{H}-8->\operatorname{LUMO}(2 \%), \mathrm{H}-3- \\
& >\text { LUMO }(2 \%)\end{aligned}$ \\
\hline 331.0041 & 0.0017 & $\begin{array}{c}\text { H-10->LUMO }(17 \%), \mathrm{H}-10->\mathrm{L}+1(10 \%), \mathrm{H}-8->\mathrm{LUMO} \\
(39 \%), \mathrm{H}-8->\mathrm{L}+1(25 \%)\end{array}$ & H-6->LUMO (3\%) \\
\hline
\end{tabular}




\begin{tabular}{|c|c|c|c|}
\hline 330.7303 & 0.0191 & $\begin{array}{c}\text { H-9->LUMO (18\%), H-9->L+1 (18\%), H-7->LUMO } \\
(12 \%), \text { H-7->L+1 (11\%), H-6->LUMO (15\%), H-6- } \\
>\text { L+1 (14\%) }\end{array}$ & H-10->L+1 (2\%), H-8->LUMO (4\%) \\
\hline 329.8768 & 0.0083 & $\begin{array}{c}\text { H-9->LUMO (29\%), H-9->L+1 (28\%), H-6->LUMO } \\
(11 \%)\end{array}$ & $\begin{array}{c}\text { H-8->LUMO }(4 \%), \mathrm{H}-8->\mathrm{L}+1(5 \%), \mathrm{H}-7->\mathrm{LUMO} \\
(2 \%), \mathrm{H}-6->\mathrm{L}+1(9 \%)\end{array}$ \\
\hline 328.4698 & 0.0365 & $\begin{array}{c}\text { H-7->LUMO }(35 \%), \text { H-7->L+1 (16\%), H-6->LUMO } \\
(26 \%), \text { H-6->L+1 (13\%) }\end{array}$ & H-12->LUMO (2\%) \\
\hline 321.8697 & 0.0077 & $\begin{array}{c}\mathrm{H}-11->\mathrm{L}+1(11 \%), \mathrm{H}-10->\mathrm{LUMO}(30 \%), \mathrm{H}-7->\mathrm{LUMO} \\
(18 \%)\end{array}$ & $\begin{array}{c}\mathrm{H}-10->\mathrm{L}+1(3 \%), \mathrm{H}-8->\mathrm{LUMO}(7 \%), \mathrm{H}-8->\mathrm{L}+1 \\
(3 \%), \mathrm{H}-7->\mathrm{L}+1(2 \%), \mathrm{H}-6->\mathrm{LUMO}(6 \%), \mathrm{H}-4- \\
>\mathrm{L}+1(4 \%), \mathrm{H}-3->\mathrm{L}+1(3 \%)\end{array}$ \\
\hline 320.9033 & 0.0012 & H-4->LUMO (30\%), H-4->L+1 (47\%) & $\begin{array}{l}\text { H-10->LUMO (4\%), H-7->LUMO (2\%), H-7->L+1 } \\
(4 \%), \text { H-5->LUMO (3\%), H-5->L+1 (2\%) }\end{array}$ \\
\hline 320.4306 & 0.0006 & H-5->LUMO (34\%), H-5->L+1 (34\%) & $\begin{array}{c}\text { H-11->LUMO (3\%), H-10->L+1 (5\%), H-7->L+1 } \\
(5 \%), \text { H-6->L+1 (5\%), H-4->LUMO (5\%), H-4- } \\
>\text { L+1 (7\%) }\end{array}$ \\
\hline 319.6787 & 0.0061 & $\begin{array}{c}\mathrm{H}-11->\mathrm{LUMO}(20 \%), \underset{(13 \%)}{\mathrm{H}-10->\mathrm{L}+1}(21 \%), \mathrm{H}-5->\mathrm{L}+1 \\
\end{array}$ & $\begin{array}{c}\text { H-10->LUMO (3\%), H-8->L+1 (6\%), H-7->L+1 } \\
\begin{array}{c}(9 \%), \mathrm{H}-6->\mathrm{L}+1(3 \%), \mathrm{H}-5->\mathrm{LUMO}(9 \%), \mathrm{H}-4- \\
>\mathrm{L}+1(4 \%)\end{array}\end{array}$ \\
\hline 314.9125 & 0.0077 & $\begin{array}{l}\text { H-12->LUMO (11\%), H-11->LUMO (15\%), H-7->L+1 } \\
\text { (30\%), H-6->LUMO (26\%) }\end{array}$ & H-12->L+1 (5\%), H-8->LUMO (3\%) \\
\hline 313.6934 & 0.0106 & $\begin{array}{c}\mathrm{H}-11->\mathrm{L}+1(15 \%), \mathrm{H}-7->\mathrm{LUMO}(18 \%), \mathrm{H}-6->\mathrm{L}+1 \\
(41 \%)\end{array}$ & $\begin{array}{l}\text { H-13->LUMO (2\%), H-12->LUMO (3\%), H-12- } \\
\quad>\mathrm{L}+1(3 \%), \mathrm{H}-8->\mathrm{L}+1(2 \%), \mathrm{H}-7->\mathrm{L}+1(8 \%)\end{array}$ \\
\hline 310.5816 & 0.0407 & H-12->LUMO (41\%), H-12->L+1 (26\%) & $\begin{array}{l}\text { H-11->LUMO (4\%), H-8->LUMO (3\%), H-7- } \\
>\text { LUMO (4\%), H-7->L+1 (4\%), H-6->L+1 (2\%) }\end{array}$ \\
\hline 308.2267 & 0.058 & H-13->LUMO (41\%), H-13->L+1 (41\%) & $\mathrm{H}-7->\mathrm{L}+1(4 \%)$ \\
\hline 300.6771 & 0.0015 & $\begin{array}{c}\text { H-11->LUMO (12\%), H-11->L+1 (10\%), H-8->LUMO } \\
(19 \%), \mathrm{H}-8->\mathrm{L}+1(43 \%)\end{array}$ & $\begin{array}{c}\text { H-10->LUMO (3\%), H-6->LUMO (5\%), H-6->L+1 } \\
(5 \%)\end{array}$ \\
\hline
\end{tabular}




\begin{tabular}{|c|c|c|c|}
\hline 298.9444 & 0.0001 & H-9->LUMO (43\%), H-9->L+1 (42\%) & $\begin{array}{c}\text { H-10->LUMO }(2 \%), \mathrm{H}-10->\mathrm{L}+1(4 \%), \mathrm{H}-7->\mathrm{LUMO} \\
(2 \%), \mathrm{H}-7->\mathrm{L}+1(2 \%)\end{array}$ \\
\hline 298.0604 & 0.001 & $\begin{array}{c}\text { H-11->LUMO (34\%), H-10->L+1 (39\%), H-8->LUMO } \\
(13 \%)\end{array}$ & H-9->LUMO (2\%), H-9->L+1 (6\%) \\
\hline 297.5311 & 0.0004 & H-11->L+1 (48\%), H-10->LUMO (30\%) & $\begin{array}{c}\mathrm{H}-10->\mathrm{L}+1(5 \%), \mathrm{H}-9->\mathrm{LUMO}(3 \%), \mathrm{H}-8->\mathrm{L}+1 \\
(8 \%)\end{array}$ \\
\hline 288.2281 & 0.0001 & H-12->LUMO (37\%), H-12->L+1 (61\%) & \\
\hline 286.5891 & 0.0002 & H-13->LUMO (49\%), H-13->L+1 (49\%) & \\
\hline 285.1195 & 0.0173 & H-1->L+2 (12\%), HOMO->L+2 (68\%) & $\begin{array}{c}\mathrm{H}-1->\mathrm{L}+6(2 \%), \mathrm{HOMO}->\mathrm{L}+3(4 \%), \mathrm{HOMO}->\mathrm{L}+6 \\
\text { (3\%), HOMO->L+7 (2\%) }\end{array}$ \\
\hline 284.2566 & 0.0223 & $\mathrm{H}-1->\mathrm{L}+3(20 \%)$, HOMO->L+3 (74\%) & HOMO->L+2 (4\%) \\
\hline 281.1497 & 0.0339 & \multicolumn{2}{|c|}{ H-1->L+4 (12\%), H-1->L+5 (22\%), HOMO->L+4 (27\%), HOMO->L+5 (38\%) } \\
\hline 279.3947 & 0.0701 & $\begin{array}{c}\mathrm{H}-1->\mathrm{L}+4(10 \%), \underset{(25 \%)}{\mathrm{HOMO}->\mathrm{L}+4}(43 \%), \mathrm{HOMO}->\mathrm{L}+5 \\
\text { (25) }\end{array}$ & $\begin{array}{c}\mathrm{H}-1->\mathrm{L}+5(7 \%), \mathrm{H}-1->\mathrm{L}+6(5 \%), \mathrm{H}-1->\mathrm{L}+7(3 \%) \\
\text { HOMO->L+7 }(4 \%)\end{array}$ \\
\hline 271.2824 & 0.0471 & $\begin{array}{c}\mathrm{H}-1->\mathrm{L}+2(43 \%), \mathrm{HOMO}->\mathrm{L}+2(16 \%), \mathrm{HOMO}->\mathrm{L}+6 \\
(29 \%)\end{array}$ & $\mathrm{H}-1->\mathrm{L}+4(6 \%)$ \\
\hline 270.0063 & 0.0089 & $\begin{array}{c}\mathrm{H}-1->\mathrm{L}+2(13 \%), \mathrm{H}-1->\mathrm{L}+4(27 \%), \mathrm{HOMO}->\mathrm{L}+4 \\
(13 \%), \mathrm{HOMO}->\mathrm{L}+7(23 \%)\end{array}$ & $\begin{array}{c}\mathrm{H}-1->\mathrm{L}+5(7 \%), \mathrm{HOMO}->\mathrm{L}+5(7 \%), \mathrm{HOMO}->\mathrm{L}+6 \\
(7 \%)\end{array}$ \\
\hline 263.4654 & 0.0003 & $\mathrm{H}-1->\mathrm{L}+3(78 \%), \mathrm{HOMO}->\mathrm{L}+3(21 \%)$ & \\
\hline 262.9792 & 0.1845 & $\begin{array}{c}\mathrm{H}-1->\mathrm{L}+4(32 \%), \underset{(28 \%)}{\mathrm{HOMO}->\mathrm{L}+5}(10 \%), \mathrm{HOMO}->\mathrm{L}+6 \\
\end{array}$ & $\begin{array}{c}\mathrm{H}-1->\mathrm{L}+2(8 \%), \mathrm{H}-1->\mathrm{L}+5(7 \%), \mathrm{H}-1->\mathrm{L}+7(3 \%) \\
\text { HOMO->L+4 }(8 \%)\end{array}$ \\
\hline
\end{tabular}




\begin{tabular}{|c|c|c|c|}
\hline 262.1286 & 0.1393 & $\begin{array}{c}\mathrm{H}-1->\mathrm{L}+2(10 \%), \mathrm{H}-1->\mathrm{L}+5(39 \%), \text { HOMO->L+5 } \\
(15 \%), \text { HOMO->L+6 (22\%) }\end{array}$ & $\begin{array}{c}\mathrm{H}-1->\mathrm{L}+7(3 \%), \mathrm{HOMO}->\mathrm{L}+4(3 \%), \mathrm{HOMO}->\mathrm{L}+7 \\
(5 \%)\end{array}$ \\
\hline 258.791 & 0.2305 & $\mathrm{H}-1->\mathrm{L}+5(13 \%), \mathrm{HOMO}->\mathrm{L}+7(60 \%)$ & $\mathrm{H}-1->\mathrm{L}+2(8 \%), \mathrm{H}-1->\mathrm{L}+4(9 \%)$ \\
\hline 254.64 & 0.1032 & $\mathrm{H}-1->\mathrm{L}+6(12 \%), \mathrm{H}-1->\mathrm{L}+7(76 \%)$ & HOMO->L+2 (3\%), HOMO->L+6 (5\%) \\
\hline 253.754 & 0.2677 & $\mathrm{H}-1->\mathrm{L}+6(76 \%), \mathrm{H}-1->\mathrm{L}+7(10 \%)$ & HOMO->L+4 (3\%), HOMO->L+5 (2\%) \\
\hline 239.6061 & 0.0303 & H-14->LUMO (67\%), H-14->L+1 (16\%) & $\begin{array}{c}\mathrm{H}-15->\mathrm{L}+1(6 \%), \mathrm{H}-1->\mathrm{L}+8(2 \%), \mathrm{HOMO}->\mathrm{L}+8 \\
(2 \%)\end{array}$ \\
\hline 238.2204 & 0.027 & H-15->LUMO (38\%), H-14->L+1 (36\%) & 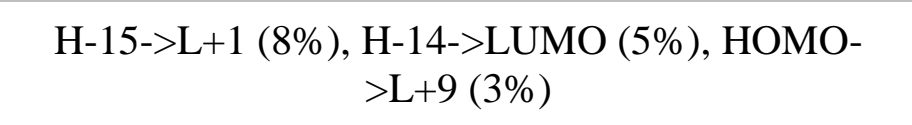 \\
\hline 234.4367 & 0.0015 & \multicolumn{2}{|c|}{ H-15->LUMO (30\%), H-15->L+1 (11\%), H-14->LUMO (19\%), H-14->L+1 (38\%) } \\
\hline 231.7635 & 0.0008 & H-15->LUMO (25\%), H-15->L+1 (67\%) & H-14->LUMO (4\%), H-14->L+1 (3\%) \\
\hline 228.3108 & 0.0013 & $\mathrm{H}-10->\mathrm{L}+2(11 \%), \mathrm{H}-8->\mathrm{L}+3(12 \%), \mathrm{H}-2->\mathrm{L}+3(28 \%)$ & $\begin{array}{c}\mathrm{H}-8->\mathrm{L}+2(8 \%), \mathrm{H}-6->\mathrm{L}+3(6 \%), \mathrm{H}-4->\mathrm{L}+2(3 \%) \\
\mathrm{H}-3->\mathrm{L}+3(8 \%), \mathrm{H}-2->\mathrm{L}+2(8 \%)\end{array}$ \\
\hline 224.4383 & 0.0006 & $\mathrm{H}-9->\mathrm{L}+5(30 \%), \mathrm{H}-3->\mathrm{L}+4(18 \%)$ & $\begin{array}{c}\mathrm{H}-11->\mathrm{L}+4(4 \%), \mathrm{H}-10->\mathrm{L}+4(6 \%), \mathrm{H}-9->\mathrm{L}+4(4 \%) \\
\mathrm{H}-8->\mathrm{L}+4(5 \%), \mathrm{H}-6->\mathrm{L}+4(2 \%), \mathrm{H}-5->\mathrm{L}+4(3 \%) \\
\mathrm{H}-5->\mathrm{L}+5(2 \%), \mathrm{H}-2->\mathrm{L}+4(8 \%)\end{array}$ \\
\hline 221.4122 & 0.0111 & $\begin{array}{c}\mathrm{H}-16->\mathrm{LUMO}(20 \%), \mathrm{H}-16->\mathrm{L}+1(13 \%), \mathrm{H}-2->\mathrm{L}+2 \\
(46 \%)\end{array}$ & $\mathrm{H}-3->\mathrm{L}+2(4 \%), \mathrm{H}-2->\mathrm{L}+3(9 \%)$ \\
\hline 220.8168 & 0.0003 & H-17->LUMO (42\%), H-17->L+1 (46\%) & H-16->LUMO (6\%), H-16->L+1 (5\%) \\
\hline 220.7892 & 0.0032 & $\begin{array}{c}\mathrm{H}-16->\mathrm{LUMO}(33 \%), \mathrm{H}-16->\mathrm{L}+1(23 \%), \mathrm{H}-2->\mathrm{L}+2 \\
(26 \%)\end{array}$ & $\begin{array}{c}\mathrm{H}-17->\mathrm{LUMO}(5 \%), \mathrm{H}-3->\mathrm{L}+2(2 \%), \mathrm{H}-2->\mathrm{L}+3 \\
(5 \%)\end{array}$ \\
\hline
\end{tabular}




\begin{tabular}{|c|c|c|c|}
\hline 216.5171 & 0.027 & $\begin{array}{c}\mathrm{H}-2->\mathrm{L}+4(12 \%), \mathrm{H}-2->\mathrm{L}+6(13 \%), \mathrm{HOMO}->\mathrm{L}+8 \\
(22 \%), \mathrm{HOMO}->\mathrm{L}+9(18 \%)\end{array}$ & $\begin{array}{c}\mathrm{H}-3->\mathrm{L}+4(3 \%), \mathrm{H}-3->\mathrm{L}+7(4 \%), \mathrm{H}-2->\mathrm{L}+3(2 \%), \\
\mathrm{H}-2->\mathrm{L}+5(4 \%), \mathrm{H}-1->\mathrm{L}+8(7 \%), \mathrm{H}-1->\mathrm{L}+9(3 \%)\end{array}$ \\
\hline 216.1736 & 0.0557 & $\begin{array}{c}\mathrm{H}-2->\mathrm{L}+7 \text { (11\%), } \mathrm{HOMO}->\mathrm{L}+8(20 \%), \mathrm{HOMO}->\mathrm{L}+9 \\
(28 \%)\end{array}$ & $\begin{array}{c}\mathrm{H}-3->\mathrm{L}+4(3 \%), \mathrm{H}-3->\mathrm{L}+6(4 \%), \mathrm{H}-2->\mathrm{L}+3(3 \%) \\
\mathrm{H}-2->\mathrm{L}+4(5 \%), \mathrm{H}-2->\mathrm{L}+6(3 \%), \mathrm{H}-1->\mathrm{L}+8(6 \%) \\
\mathrm{H}-1->\mathrm{L}+9(5 \%)\end{array}$ \\
\hline 215.8048 & 0.0162 & $\mathrm{H}-3->\mathrm{L}+5(41 \%), \mathrm{H}-2->\mathrm{L}+5(49 \%)$ & HOMO->L+9 (3\%) \\
\hline 214.6318 & 0.0079 & $\mathrm{H}-4->\mathrm{L}+2(12 \%), \mathrm{H}-2->\mathrm{L}+3(40 \%)$ & $\begin{array}{c}\mathrm{H}-11->\mathrm{L}+3(3 \%), \mathrm{H}-10->\mathrm{L}+2(2 \%), \mathrm{H}-8->\mathrm{L}+2(2 \%) \\
\mathrm{H}-8->\mathrm{L}+3(8 \%), \mathrm{H}-6->\mathrm{L}+3(4 \%), \mathrm{H}-4->\mathrm{L}+3(5 \%) \\
\mathrm{H}-2->\mathrm{L}+2(5 \%), \mathrm{HOMO}->\mathrm{L}+9(9 \%)\end{array}$ \\
\hline 213.7585 & 0.0301 & $\mathrm{H}-2->\mathrm{L}+4(41 \%), \mathrm{HOMO}->\mathrm{L}+8(25 \%)$ & $\begin{array}{c}\mathrm{H}-9->\mathrm{L}+5(3 \%), \mathrm{H}-5->\mathrm{L}+4(3 \%), \mathrm{H}-3->\mathrm{L}+4(8 \%), \\
\mathrm{H}-1->\mathrm{L}+8(3 \%)\end{array}$ \\
\hline 211.3535 & 0.0245 & $\mathrm{H}-4->\mathrm{L}+2(70 \%), \mathrm{H}-4->\mathrm{L}+3(13 \%)$ & H-10->L+2 (2\%), HOMO->L+9 (3\%) \\
\hline 211.1843 & 0.0195 & $\mathrm{H}-3->\mathrm{L}+2(27 \%), \mathrm{HOMO}->\mathrm{L}+10(28 \%)$ & $\begin{array}{c}\mathrm{H}-3->\mathrm{L}+4(6 \%), \mathrm{H}-3->\mathrm{L}+6(2 \%), \mathrm{H}-3->\mathrm{L}+7(9 \%), \\
\mathrm{H}-2->\mathrm{L}+2(6 \%), \mathrm{H}-2->\mathrm{L}+7(3 \%)\end{array}$ \\
\hline 210.6032 & 0.0034 & $\mathrm{H}-3->\mathrm{L}+4(25 \%), \mathrm{H}-2->\mathrm{L}+4(10 \%), \mathrm{H}-2->\mathrm{L}+6(17 \%)$ & $\begin{array}{c}\mathrm{H}-3->\mathrm{L}+2(6 \%), \mathrm{H}-3->\mathrm{L}+5(8 \%), \mathrm{H}-3->\mathrm{L}+6(5 \%) \\
\mathrm{H}-2->\mathrm{L}+5(6 \%), \mathrm{H}-1->\mathrm{L}+10(2 \%), \mathrm{HOMO}->\mathrm{L}+9 \\
(6 \%)\end{array}$ \\
\hline 210.296 & 0.0159 & H-3->L+2 (33\%), H-2->L+6 (18\%), H-2->L+7 (12\%) & $\mathrm{H}-4->\mathrm{L}+3(8 \%), \mathrm{H}-2->\mathrm{L}+4(3 \%), \mathrm{HOMO}->\mathrm{L}+9(9 \%)$ \\
\hline 209.1678 & 0.006 & $\mathrm{H}-3->\mathrm{L}+6(10 \%), \mathrm{H}-2->\mathrm{L}+7(28 \%)$ & $\begin{array}{c}\mathrm{H}-5->\mathrm{L}+4(6 \%), \mathrm{H}-5->\mathrm{L}+5(5 \%), \mathrm{H}-3->\mathrm{L}+7(7 \%) \\
\mathrm{H}-2->\mathrm{L}+6(5 \%), \mathrm{H}-1->\mathrm{L}+8(4 \%), \mathrm{H}-1->\mathrm{L}+10(4 \%) \\
\text { HOMO->L+8 }(9 \%), \mathrm{HOMO}->\mathrm{L}+10(5 \%)\end{array}$ \\
\hline 208.8084 & 0.0087 & $\mathrm{H}-4->\mathrm{L}+3(59 \%)$ & $\begin{array}{c}\mathrm{H}-11->\mathrm{L}+3(2 \%), \mathrm{H}-10->\mathrm{L}+2(2 \%), \mathrm{H}-4->\mathrm{L}+2(6 \%) \\
\mathrm{H}-3->\mathrm{L}+3(7 \%), \mathrm{H}-2->\mathrm{L}+3(3 \%), \mathrm{H}-2->\mathrm{L}+6(3 \%)\end{array}$ \\
\hline 208.0132 & 0.0011 & $\mathrm{H}-5->\mathrm{L}+4(16 \%), \mathrm{H}-5->\mathrm{L}+5(57 \%)$ & $\begin{array}{c}\mathrm{H}-9->\mathrm{L}+5(2 \%), \mathrm{H}-3->\mathrm{L}+4(3 \%), \mathrm{H}-1->\mathrm{L}+8(3 \%), \\
\text { HOMO->L+10 }(4 \%)\end{array}$ \\
\hline
\end{tabular}




\begin{tabular}{|c|c|c|c|}
\hline 207.9121 & 0.0246 & $\begin{array}{c}\mathrm{H}-5->\mathrm{L}+5(24 \%), \mathrm{H}-1->\mathrm{L}+8(13 \%), \mathrm{HOMO}->\mathrm{L}+10 \\
(11 \%)\end{array}$ & $\begin{array}{c}\mathrm{H}-5->\mathrm{L}+4(8 \%), \mathrm{H}-3->\mathrm{L}+2(5 \%), \mathrm{H}-3->\mathrm{L}+4(5 \%) \\
\mathrm{H}-3->\mathrm{L}+7(2 \%), \mathrm{H}-2->\mathrm{L}+4(4 \%), \mathrm{H}-2->\mathrm{L}+6(5 \%) \\
\text { HOMO->L+8 }(5 \%)\end{array}$ \\
\hline 207.5362 & 0.003 & $\begin{array}{c}\text { H-19->LUMO (18\%), H-19->L+1 (18\%), H-5->L+4 } \\
\text { (21\%), H-1->L+8 (19\%) }\end{array}$ & $\begin{array}{c}\mathrm{H}-3->\mathrm{L}+5(3 \%), \mathrm{H}-2->\mathrm{L}+5(3 \%), \mathrm{H}-2->\mathrm{L}+7(3 \%) \\
\mathrm{HOMO}->\mathrm{L}+8(6 \%)\end{array}$ \\
\hline 207.134 & 0.0044 & $\begin{array}{c}\mathrm{H}-19->\mathrm{LUMO}(27 \%), \mathrm{H}-19->\mathrm{L}+1(28 \%), \mathrm{H}-5->\mathrm{L}+4 \\
(13 \%)\end{array}$ & $\mathrm{H}-5->\mathrm{L}+5(4 \%), \mathrm{H}-2->\mathrm{L}+7(4 \%), \mathrm{H}-1->\mathrm{L}+8(9 \%)$ \\
\hline 206.6093 & 0.0057 & $\begin{array}{c}\mathrm{H}-3->\mathrm{L}+5(17 \%), \mathrm{H}-2->\mathrm{L}+5(13 \%), \mathrm{H}-1->\mathrm{L}+8(15 \%) \\
\text { HOMO->L+10 }(11 \%)\end{array}$ & $\begin{array}{c}\mathrm{H}-7->\mathrm{L}+2(3 \%), \mathrm{H}-3->\mathrm{L}+2(4 \%), \mathrm{H}-3->\mathrm{L}+3(2 \%) \\
\mathrm{H}-3->\mathrm{L}+7(3 \%), \mathrm{H}-2->\mathrm{L}+6(8 \%), \mathrm{H}-1->\mathrm{L}+10(3 \%) \\
\text { HOMO->L+8 }(2 \%)\end{array}$ \\
\hline 206.4545 & 0.004 & $\begin{array}{c}\mathrm{H}-5->\mathrm{L}+4(17 \%), \mathrm{H}-3->\mathrm{L}+4(14 \%), \mathrm{H}-3->\mathrm{L}+5(18 \%), \\
\mathrm{H}-2->\mathrm{L}+5(17 \%)\end{array}$ & $\begin{array}{c}\mathrm{H}-2->\mathrm{L}+4(5 \%), \mathrm{H}-1->\mathrm{L}+8(9 \%), \mathrm{HOMO}->\mathrm{L}+10 \\
(2 \%)\end{array}$ \\
\hline 205.6327 & 0.0024 & $\mathrm{H}-3->\mathrm{L}+3(70 \%)$ & $\begin{array}{c}\mathrm{H}-6->\mathrm{L}+3(2 \%), \mathrm{H}-4->\mathrm{L}+3(3 \%), \mathrm{H}-2->\mathrm{L}+3(4 \%), \\
\mathrm{H}-1->\mathrm{L}+9(7 \%)\end{array}$ \\
\hline 205.2481 & 0.0025 & $\mathrm{H}-1->\mathrm{L}+9(59 \%)$ & $\begin{array}{c}\text { H-20->LUMO (9\%), H-20->L+1 (5\%), H-3->L+3 } \\
(6 \%), \text { HOMO->L+9 (8\%) }\end{array}$ \\
\hline 205.0274 & 0.0154 & $\begin{array}{c}\mathrm{H}-20->\mathrm{LUMO}(48 \%), \mathrm{H}-20->\mathrm{L}+1(30 \%), \mathrm{H}-1->\mathrm{L}+9 \\
(12 \%)\end{array}$ & H-18->LUMO (2\%) \\
\hline 203.6701 & 0.0019 & H-21->LUMO (30\%), H-21->L+1 (32\%) & $\mathrm{H}-2->\mathrm{L}+7(6 \%), \mathrm{H}-1->\mathrm{L}+10(9 \%)$ \\
\hline 203.5364 & 0.0044 & $\begin{array}{c}\text { H-21->LUMO (16\%), H-21->L+1 (17\%), H-2->L+7 } \\
(10 \%), \mathrm{H}-1->\mathrm{L}+10(16 \%)\end{array}$ & $\begin{array}{c}\mathrm{H}-7->\mathrm{L}+2(3 \%), \mathrm{H}-7->\mathrm{L}+7(3 \%), \mathrm{H}-6->\mathrm{L}+2(3 \%), \\
\mathrm{H}-6->\mathrm{L}+6(3 \%), \mathrm{H}-3->\mathrm{L}+4(4 \%), \mathrm{H}-2->\mathrm{L}+6(2 \%)\end{array}$ \\
\hline 202.367 & 0.0021 & $\mathrm{H}-3->\mathrm{L}+6(38 \%), \mathrm{H}-3->\mathrm{L}+7(15 \%), \mathrm{H}-2->\mathrm{L}+7(12 \%)$ & $\begin{array}{c}\mathrm{H}-5->\mathrm{L}+7(3 \%), \mathrm{H}-2->\mathrm{L}+4(3 \%), \mathrm{H}-2->\mathrm{L}+5(2 \%) \\
\mathrm{H}-2->\mathrm{L}+6(5 \%), \mathrm{H}-1->\mathrm{L}+10(6 \%)\end{array}$ \\
\hline 201.8465 & 0.0022 & $\begin{array}{c}\mathrm{H}-22->\mathrm{LUMO}(39 \%), \mathrm{H}-22->\mathrm{L}+1(27 \%), \mathrm{H}-3->\mathrm{L}+7 \\
(12 \%)\end{array}$ & $\mathrm{H}-3->\mathrm{L}+6(7 \%)$ \\
\hline 201.7709 & 0.0038 & $\begin{array}{c}\text { H-22->LUMO (15\%), H-22->L+1 (10\%), H-3->L+6 } \\
(17 \%), \mathrm{H}-3->\mathrm{L}+7(31 \%)\end{array}$ & $\begin{array}{c}\mathrm{H}-4->\mathrm{L}+6(5 \%), \mathrm{H}-4->\mathrm{L}+7(3 \%), \mathrm{H}-3->\mathrm{L}+2(5 \%), \\
\mathrm{H}-2->\mathrm{L}+6(4 \%)\end{array}$ \\
\hline
\end{tabular}




\begin{tabular}{|c|c|c|c|}
\hline 201.3744 & 0.0011 & H-18->LUMO (61\%), H-17->LUMO (17\%) & $\begin{array}{c}\mathrm{H}-19->\mathrm{LUMO}(2 \%), \mathrm{H}-17->\mathrm{L}+1(4 \%), \mathrm{H}-16->\mathrm{L}+1 \\
(9 \%)\end{array}$ \\
\hline 200.8199 & 0.0007 & H-4->L+6 (38\%), H-4->L+7 (19\%) & $\begin{array}{c}\text { H-17->LUMO (2\%), H-17->L+1 (8\%), H-16- } \\
\text { >LUMO (4\%), H-3->L+6 (3\%), H-3->L+7 (3\%), } \\
\text { HOMO->L+13 (2\%) }\end{array}$ \\
\hline 200.7744 & 0.0008 & $\begin{array}{c}\text { H-17->LUMO }(13 \%), \mathrm{H}-17->\mathrm{L}+1(35 \%), \mathrm{H}-16- \\
\text { >LUMO (19\%) }\end{array}$ & $\begin{array}{c}\text { H-18->LUMO (4\%), H-18->L+1 (6\%), H-16->L+1 } \\
\text { (3\%), H-4->L+6 (9\%), H-4->L+7 (4\%) }\end{array}$ \\
\hline 200.3526 & 0.0004 & $\begin{array}{l}\text { H-18-> LUMO }(22 \%), \text { H-17->LUMO (16\%), H-16- } \\
\text { >LUMO (11\%), H-16->L+1 (40\%) }\end{array}$ & $\mathrm{H}-5->\mathrm{L}+6(3 \%), \mathrm{H}-5->\mathrm{L}+7(3 \%)$ \\
\hline 200.3073 & 0.0045 & $\mathrm{H}-5->\mathrm{L}+2(11 \%), \mathrm{H}-5->\mathrm{L}+6(32 \%), \mathrm{H}-5->\mathrm{L}+7(31 \%)$ & $\begin{array}{c}\mathrm{H}-18->\mathrm{LUMO}(2 \%), \mathrm{H}-16->\mathrm{L}+1(3 \%), \mathrm{H}-3->\mathrm{L}+7 \\
(3 \%)\end{array}$ \\
\hline 199.6139 & 0.0016 & $\mathrm{H}-18->\mathrm{L}+1(79 \%)$ & H-19->L+1 (2\%), H-16->LUMO (5\%) \\
\hline 199.5079 & 0.0054 & H-6->L+2 (25\%), H-5->L+2 (25\%) & $\begin{array}{c}\mathrm{H}-18->\mathrm{L}+1(4 \%), \mathrm{H}-10->\mathrm{L}+3(6 \%), \mathrm{H}-8->\mathrm{L}+2(7 \%) \\
\mathrm{H}-8->\mathrm{L}+3(3 \%), \mathrm{H}-5->\mathrm{L}+7(7 \%), \mathrm{H}-1->\mathrm{L}+10(3 \%)\end{array}$ \\
\hline 199.3283 & 0.0044 & $\mathrm{H}-5->\mathrm{L}+2(45 \%)$ & $\begin{array}{c}\mathrm{H}-10->\mathrm{L}+3(5 \%), \mathrm{H}-8->\mathrm{L}+2(7 \%), \mathrm{H}-8->\mathrm{L}+3(2 \%) \\
\mathrm{H}-7->\mathrm{L}+2(6 \%), \mathrm{H}-6->\mathrm{L}+2(4 \%), \mathrm{H}-5->\mathrm{L}+7(6 \%) \\
\mathrm{H}-1->\mathrm{L}+10(3 \%), \mathrm{HOMO}->\mathrm{L}+10(2 \%)\end{array}$ \\
\hline 198.4287 & 0.0122 & $\mathrm{H}-7->\mathrm{L}+2(30 \%), \mathrm{H}-4->\mathrm{L}+4(15 \%)$ & $\begin{array}{c}\mathrm{H}-12->\mathrm{L}+2(5 \%), \mathrm{H}-10->\mathrm{L}+2(3 \%), \mathrm{H}-8->\mathrm{L}+2(3 \%), \\
\mathrm{H}-7->\mathrm{L}+4(2 \%), \mathrm{H}-7->\mathrm{L}+6(4 \%), \mathrm{H}-6->\mathrm{L}+2(2 \%), \\
\mathrm{H}-5->\mathrm{L}+2(7 \%), \mathrm{H}-4->\mathrm{L}+5(3 \%), \mathrm{H}-1->\mathrm{L}+11(3 \%), \\
\text { HOMO->L+10 (7\%) }\end{array}$ \\
\hline 198.1401 & 0.0023 & $\mathrm{H}-4->\mathrm{L}+4(58 \%)$ & $\mathrm{H}-7->\mathrm{L}+2(8 \%), \mathrm{H}-4->\mathrm{L}+5(8 \%), \mathrm{H}-4->\mathrm{L}+7(7 \%)$ \\
\hline 197.6915 & 0.0017 & H-9->L+4 (31\%), H-6->L+5 (10\%) & $\begin{array}{c}\mathrm{H}-11->\mathrm{L}+5(5 \%), \mathrm{H}-10->\mathrm{L}+5(9 \%), \mathrm{H}-9->\mathrm{L}+5(4 \%) \\
\mathrm{H}-8->\mathrm{L}+5(9 \%), \mathrm{H}-7->\mathrm{L}+4(6 \%), \mathrm{H}-1->\mathrm{L}+10(7 \%) \\
\text { HOMO->L+11 }(6 \%)\end{array}$ \\
\hline 197.3673 & 0.007 & H-1->L+10 (20\%), HOMO->L+11 (22\%) & $\begin{array}{c}\mathrm{H}-11->\mathrm{L}+2(3 \%), \mathrm{H}-10->\mathrm{L}+3(2 \%), \mathrm{H}-10->\mathrm{L}+5 \\
(2 \%), \mathrm{H}-9->\mathrm{L}+4(7 \%), \mathrm{H}-8->\mathrm{L}+2(5 \%), \mathrm{H}-8->\mathrm{L}+3\end{array}$ \\
\hline
\end{tabular}




\begin{tabular}{|c|c|c|c|}
\hline & & & $\begin{array}{c}(2 \%), \mathrm{H}-7->\mathrm{L}+4(3 \%), \mathrm{H}-6->\mathrm{L}+2(2 \%), \mathrm{H}-6->\mathrm{L}+4 \\
(8 \%), \mathrm{H}-6->\mathrm{L}+6(6 \%)\end{array}$ \\
\hline 196.991 & 0.0275 & H-7->L+4 (11\%), H-6->L+2 (14\%) & $\begin{array}{c}\text { H-11->L+2 }(8 \%), \mathrm{H}-8->\mathrm{L}+2(6 \%), \mathrm{H}-8->\mathrm{L}+3(2 \%), \\
\mathrm{H}-7->\mathrm{L}+2(2 \%), \mathrm{H}-7->\mathrm{L}+5(4 \%), \mathrm{H}-7->\mathrm{L}+7(3 \%), \\
\text { H-6->L+4 }(4 \%), \mathrm{H}-6->\mathrm{L}+6(4 \%), \mathrm{H}-6->\mathrm{L}+7(6 \%), \\
\text { H-1->L+10 (5\%), H-1->L+11 (4\%), HOMO->L+10 } \\
(5 \%), \text { HOMO->L+11 (4\%) }\end{array}$ \\
\hline 196.9472 & 0 & H-5->L+3 (99\%) & \\
\hline 196.7472 & 0.0146 & $\mathrm{H}-12->\mathrm{L}+2(24 \%), \mathrm{H}-12->\mathrm{L}+3(15 \%)$ & $\begin{array}{c}\mathrm{H}-11->\mathrm{L}+2(4 \%), \mathrm{H}-8->\mathrm{L}+2(7 \%), \mathrm{H}-7->\mathrm{L}+3(8 \%) \\
\mathrm{H}-7->\mathrm{L}+4(4 \%), \mathrm{H}-7->\mathrm{L}+6(7 \%), \mathrm{H}-6->\mathrm{L}+2(5 \%), \\
\mathrm{H}-6->\mathrm{L}+3(2 \%)\end{array}$ \\
\hline 196.2117 & 0.0092 & H-7->L+4 (12\%), H-7->L+5 (10\%), H-6->L+4 (18\%) & $\begin{array}{c}\text { H-12->L+3 (5\%), H-11->L+4 (2\%), H-8->L+4 (3\%), } \\
\text { H-8->L+6 }(2 \%), \text { H-8->L+7 }(2 \%), \mathrm{H}-7->\mathrm{L}+3(4 \%), \\
\text { H-7->L+7 (5\%), H-6->L+7 (7\%), H-4->L+4 (5\%), } \\
\text { H-4->L+5 (3\%) }\end{array}$ \\
\hline 195.8769 & 0.0005 & H-4->L+4 (12\%), H-4->L+5 (80\%) & \\
\hline 195.5032 & 0.0191 & H-12->L+2 (20\%), H-12->L+3 (20\%), H-7->L+3 (19\%) & $\begin{array}{c}\mathrm{H}-11->\mathrm{L}+3(4 \%), \mathrm{H}-10->\mathrm{L}+3(5 \%), \mathrm{H}-8->\mathrm{L}+3(2 \%) \\
\mathrm{H}-6->\mathrm{L}+4(3 \%), \mathrm{H}-6->\mathrm{L}+7(3 \%), \mathrm{H}-1->\mathrm{L}+11(2 \%) \\
\text { HOMO->L+11 (4\%) }\end{array}$ \\
\hline 194.9652 & 0.0001 & H-19->LUMO (49\%), H-19->L+1 (49\%) & \\
\hline 194.8732 & 0.0021 & H-13->L+5 (25\%), H-7->L+5 (11\%), H-6->L+5 (23\%) & $\begin{array}{c}\mathrm{H}-13->\mathrm{L}+4(4 \%), \mathrm{H}-11->\mathrm{L}+5(6 \%), \mathrm{H}-10->\mathrm{L}+5 \\
(6 \%), \mathrm{H}-9->\mathrm{L}+4(5 \%), \mathrm{H}-7->\mathrm{L}+4(8 \%)\end{array}$ \\
\hline 194.1774 & 0.0062 & H-13->L+4 (13\%), H-10->L+4 (11\%), H-6->L+4 (14\%) & $\begin{array}{c}\text { H-11->L+4 (6\%), H-11->L+7 (3\%), H-10->L+6 } \\
(3 \%), \mathrm{H}-10->\mathrm{L}+7(3 \%), \mathrm{H}-8->\mathrm{L}+4(3 \%), \mathrm{H}-8->\mathrm{L}+6 \\
(4 \%), \mathrm{H}-8->\mathrm{L}+7(2 \%), \mathrm{H}-7->\mathrm{L}+6(4 \%), \mathrm{H}-7->\mathrm{L}+7 \\
(3 \%), \mathrm{H}-6->\mathrm{L}+7(4 \%), \mathrm{H}-1->\mathrm{L}+11(2 \%), \mathrm{HOMO}- \\
>\mathrm{L}+11(3 \%)\end{array}$ \\
\hline
\end{tabular}




\begin{tabular}{|c|c|c|c|}
\hline 193.4804 & 0.0135 & $\mathrm{H}-10->\mathrm{L}+2(24 \%), \mathrm{H}-10->\mathrm{L}+6(17 \%)$ & $\begin{array}{c}\mathrm{H}-12->\mathrm{L}+2(6 \%), \mathrm{H}-11->\mathrm{L}+2(5 \%), \mathrm{H}-11->\mathrm{L}+7 \\
(2 \%), \mathrm{H}-10->\mathrm{L}+7(4 \%), \mathrm{H}-9->\mathrm{L}+7(2 \%), \mathrm{H}-8->\mathrm{L}+6 \\
(6 \%), \mathrm{H}-8->\mathrm{L}+7(6 \%), \mathrm{H}-7->\mathrm{L}+6(3 \%), \mathrm{H}-6->\mathrm{L}+2 \\
(3 \%)\end{array}$ \\
\hline 193.3748 & 0.0247 & $\mathrm{H}-8->\mathrm{L}+6(13 \%)$ & $\begin{array}{c}\text { H-25->LUMO (4\%), H-25->L+1 (4\%), H-24- } \\
\text { >LUMO (3\%), H-24->L+1 (3\%), H-13->L+4 (7\%), } \\
\text { H-12->L+2 (7\%), H-10->L+7 (3\%), H-9->L+4 (3\%), } \\
\text { H-9->L+6 (8\%), H-9->L+7 (7\%), H-8->L+2 (6\%), } \\
\text { H-8->L+5 (3\%), H-8->L+7 (5\%) }\end{array}$ \\
\hline 193.3115 & 0.0043 & H-12->L+2 (16\%), H-8->L+6 (10\%) & $\begin{array}{c}\text { H-25->LUMO (2\%), H-25->L+1 (5\%), H-24- } \\
\text { >LUMO (4\%), H-11->L+2 (2\%), H-11->L+6 (2\%), } \\
\text { H-10->L+3 (2\%), H-9->L+6 (4\%), H-9->L+7 (4\%), } \\
\text { H-8->L+2 (4\%), H-8->L+7 (6\%), H-6->L+6 }(6 \%), \\
\text { H-6->L+7 (2\%) }\end{array}$ \\
\hline 192.7615 & 0.0257 & & $\begin{array}{c}\text { H-27->L+1 (4\%), H-26->LUMO (8\%), H-26->L+1 } \\
(4 \%), \mathrm{H}-25->\mathrm{LUMO}(7 \%), \mathrm{H}-24->\mathrm{L}+1(7 \%), \mathrm{H}-13- \\
>\mathrm{L}+4(6 \%), \mathrm{H}-13->\mathrm{L}+5(2 \%), \mathrm{H}-12->\mathrm{L}+3(9 \%), \mathrm{H}- \\
11->\mathrm{L}+3(2 \%), \mathrm{H}-11->\mathrm{L}+6(4 \%), \mathrm{H}-10->\mathrm{L}+3(4 \%), \\
\mathrm{H}-10->\mathrm{L}+7(5 \%), \mathrm{H}-8->\mathrm{L}+7(3 \%), \mathrm{H}-7->\mathrm{L}+3(5 \%), \\
\text { H-7->L+4 (3\%), H-6->L+3 (3\%), HOMO->L+11 } \\
(3 \%)\end{array}$ \\
\hline 192.6656 & 0.0062 & H-12->L+3 (29\%), H-6->L+3 (12\%) & $\begin{array}{c}\text { H-26->L+1 (3\%), H-24->LUMO (3\%), H-13->L+4 } \\
(3 \%), \mathrm{H}-11->\mathrm{L}+3(6 \%), \mathrm{H}-10->\mathrm{L}+3(6 \%), \mathrm{H}-8->\mathrm{L}+3 \\
(3 \%), \mathrm{H}-7->\mathrm{L}+3(8 \%), \mathrm{H}-1->\mathrm{L}+11(3 \%)\end{array}$ \\
\hline
\end{tabular}


Table S7: Td-DFT transitions data of compound 1-Cu

\begin{tabular}{|c|c|c|c|}
\hline $\begin{array}{l}\text { Wavelength } \\
\quad(\mathrm{nm})\end{array}$ & $\begin{array}{l}\text { Osc. } \\
\text { Strength }\end{array}$ & Major contribs & Minor contribs \\
\hline 778.4529 & 0.0141 & HOMO->L+1 (94\%) & $\mathrm{H}-3->\mathrm{L}+2(3 \%)$ \\
\hline 737.6938 & 0.0128 & HOMO->L+2 (89\%) & $\mathrm{H}-3->\mathrm{L}+1(5 \%)$ \\
\hline 720.1266 & 0.0086 & H-9->LUMO (86\%) & H-12->LUMO (3\%), H-6->LUMO (3\%) \\
\hline 698.7781 & 0.012 & H-13->LUMO (14\%), H-10->LUMO (73\%) & $\mathrm{H}-3->\mathrm{L}+1(2 \%)$ \\
\hline 668.3063 & 0.0001 & $\begin{array}{c}\text { H-12->LUMO (18\%), H-11->LUMO (22\%), HOMO- } \\
>\mathrm{L}+4(46 \%)\end{array}$ & H-34->LUMO (2\%), H-7->LUMO (5\%) \\
\hline 668.1623 & 0.0013 & HOMO->L+3 (87\%) & $\mathrm{H}-3->\mathrm{L}+4(2 \%)$ \\
\hline 645.6501 & 0 & $\begin{array}{c}\text { H-12->LUMO (17\%), H-11->LUMO (20\%), HOMO- } \\
>\mathrm{L}+4(45 \%)\end{array}$ & H-34->LUMO (3\%), H-7->LUMO (4\%), H-2->L+1 (4\%) \\
\hline 616.6834 & 0.0024 & $\begin{array}{c}\text { H-33->LUMO (16\%), H-19->LUMO (10\%), H-14- } \\
>\text { LUMO (44\%) }\end{array}$ & $\begin{array}{c}\text { H-39->LUMO (4\%), H-36->LUMO (3\%), H-21->LUMO } \\
\text { (5\%), H-13->LUMO (4\%), H-1->L+1 (4\%) }\end{array}$ \\
\hline 600.9024 & 0 & $\begin{array}{c}\text { H-38->LUMO (15\%), H-34->LUMO (15\%), H-24- } \\
>\text { LUMO (10\%), H-22->LUMO (33\%) }\end{array}$ & $\begin{array}{c}\text { H-35-> LUMO (7\%), H-25->LUMO (3\%), H-17->LUMO } \\
\text { (2\%), H-16->LUMO (2\%), H-2->L+1 (3\%) }\end{array}$ \\
\hline 597.1113 & 0.0177 & H-14->LUMO (15\%), H-1->L+1 (28\%) & $\begin{array}{c}\text { H-39->LUMO (8\%), H-36->LUMO (5\%), H-33->LUMO } \\
\text { (5\%), H-23->LUMO (3\%), H-21->LUMO (5\%), H-20- } \\
\text { >LUMO (8\%), H-19->LUMO (9\%), H-8->LUMO (2\%), } \\
\text { H-2->L+2 (3\%) }\end{array}$ \\
\hline 585.1347 & 0.0041 & H-15->LUMO (15\%), H-3->L+1 (49\%) & $\begin{array}{c}\text { H-14->LUMO (5\%), H-13->LUMO (3\%), H-4->L+2 (4\%), } \\
\text { H-2->L+3 (4\%), H-1->L+1 (4\%), H-1->L+4 (6\%), HOMO- } \\
>\text { L+2 (5\%) }\end{array}$ \\
\hline
\end{tabular}




\begin{tabular}{|c|c|c|c|}
\hline 579.9074 & 0.0783 & $\begin{array}{c}\text { H-15->LUMO (27\%), H-13->LUMO (10\%), H-1- } \\
\text { >L+1 (27\%) }\end{array}$ & $\begin{array}{c}\text { H-21->LUMO (4\%), H-20->LUMO (2\%), H-19->LUMO } \\
(2 \%), \mathrm{H}-14->\text { LUMO }(5 \%), \mathrm{H}-3->\mathrm{L}+4(2 \%), \mathrm{H}-2->\mathrm{L}+3 \\
(3 \%), \mathrm{H}-1->\mathrm{L}+4(6 \%)\end{array}$ \\
\hline 575.7334 & 0.0252 & $\begin{array}{c}\text { H-16->LUMO (10\%), H-4->L+1 (14\%), H-3->L+2 } \\
\text { (23\%), H-1->L+2 (10\%), H-1->L+3 (15\%) }\end{array}$ & $\begin{array}{c}\text { H-24->LUMO (3\%), H-12->LUMO (4\%), H-2->L+1 (7\%), } \\
\text { H-2->L+4 (6\%) }\end{array}$ \\
\hline 575.5731 & 0.0748 & $\begin{array}{c}\text { H-15->LUMO (24\%), H-14->LUMO (11\%), H-3- } \\
>\text { L+1 (17\%), H-1->L+1 (20\%) }\end{array}$ & $\begin{array}{c}\text { H-13->LUMO }(3 \%), \mathrm{H}-4->\mathrm{L}+3(3 \%), \mathrm{H}-3->\mathrm{L}+4(7 \%), \mathrm{H}- \\
2->\mathrm{L}+3(5 \%), \mathrm{H}-1->\mathrm{L}+4(4 \%)\end{array}$ \\
\hline 575.4395 & 0.0148 & H-16->LUMO (38\%), H-1->L+3 (12\%) & $\begin{array}{c}\text { H-25->LUMO (3\%), H-24->LUMO (3\%), H-22->LUMO } \\
(7 \%), \text { H-17->LUMO (2\%), H-12->LUMO (5\%), H-11- } \\
>\text { LUMO (8\%), H-6->LUMO (2\%), H-2->L+1 (3\%), H-2- } \\
>\text { L+4 (5\%), H-1->L+2 (3\%) }\end{array}$ \\
\hline 569.1003 & 0.0022 & H-3->L+3 (23\%), H-2->L+1 (43\%) & $\begin{array}{c}\mathrm{H}-4->\mathrm{L}+1(5 \%), \mathrm{H}-4->\mathrm{L}+4(8 \%), \mathrm{H}-3->\mathrm{L}+2(9 \%), \mathrm{H}-2- \\
>\mathrm{L}+4(2 \%), \mathrm{H}-1->\mathrm{L}+3(3 \%)\end{array}$ \\
\hline 558.6887 & 0.0003 & $\mathrm{H}-2->\mathrm{L}+1(25 \%), \mathrm{H}-1->\mathrm{L}+2(60 \%)$ & $\mathrm{H}-3->\mathrm{L}+2(3 \%), \mathrm{H}-3->\mathrm{L}+3(8 \%)$ \\
\hline 551.1633 & 0.0006 & $\mathrm{H}-2->\mathrm{L}+2(75 \%)$ & $\mathrm{H}-4->\mathrm{L}+3(7 \%), \mathrm{H}-3->\mathrm{L}+4(7 \%), \mathrm{H}-1->\mathrm{L}+1(8 \%)$ \\
\hline 541.0613 & 0 & H-18->LUMO (12\%), H-17->LUMO (61\%) & $\begin{array}{c}\text { H-25->LUMO (5\%), H-24->LUMO (9\%), H-4->L+1 (3\%), } \\
\text { H-3->L+2 (3\%) }\end{array}$ \\
\hline 536.1942 & 0.0003 & $\begin{array}{c}\mathrm{H}-17->\mathrm{LUMO}(10 \%), \mathrm{H}-4->\mathrm{L}+1(42 \%), \mathrm{H}-3->\mathrm{L}+2 \\
(30 \%)\end{array}$ & $\begin{array}{c}\text { H-25->LUMO (2\%), H-22->LUMO (3\%), H-18->LUMO } \\
(3 \%), \mathrm{H}-2->\mathrm{L}+4(5 \%)\end{array}$ \\
\hline 534.8757 & 0.0001 & $\begin{array}{c}\text { H-24->LUMO (10\%), H-22->LUMO (16\%), H-18- } \\
>\text { LUMO (40\%) }\end{array}$ & $\begin{array}{c}\text { H-34->LUMO (3\%), H-27->LUMO (3\%), H-25->LUMO } \\
(4 \%), \text { H-17->LUMO (3\%), H-16->LUMO (3\%), H-4->L+1 } \\
(8 \%), \text { H-3->L+2 (5\%) }\end{array}$ \\
\hline 533.1048 & 0.0082 & $\begin{array}{l}\text { H-21->LUMO (12\%), H-19->LUMO (10\%), H-4- } \\
>\text { L+2 (30\%), H-3->L+1 (13\%), H-2->L+3 (18\%) }\end{array}$ & H-23->LUMO (3\%), H-15->LUMO (5\%) \\
\hline 531.733 & 0.0038 & $\begin{array}{c}\text { H-21->LUMO (30\%), H-19->LUMO (19\%), H-4- } \\
\text { >L+2 (16\%) }\end{array}$ & $\begin{array}{c}\text { H-29->LUMO (2\%), H-23->LUMO (6\%), H-20->LUMO } \\
(4 \%), \mathrm{H}-13->\text { LUMO (2\%), H-3->L+1 (6\%), H-2->L+3 } \\
(9 \%)\end{array}$ \\
\hline 521.4459 & 0.0703 & $\begin{array}{c}\text { H-30->LUMO (11\%), H-20->LUMO (13\%), H-2- } \\
>\text { L }+3(11 \%), \mathrm{H}-1->\mathrm{L}+4(31 \%)\end{array}$ & $\begin{array}{l}\text { H-33->LUMO (5\%), H-26->LUMO (7\%), H-23->LUMO } \\
\text { (4\%), H-21->LUMO (6\%), H-19->LUMO (3\%) }\end{array}$ \\
\hline 520.9201 & 0.0019 & $\begin{array}{l}\mathrm{H}-20->\mathrm{LUMO}(12 \%), \mathrm{H}-2->\mathrm{L}+3(16 \%), \mathrm{H}-1->\mathrm{L}+4 \\
(35 \%)\end{array}$ & $\begin{array}{c}\text { H-33->LUMO (5\%), H-30->LUMO (7\%), H-26->LUMO } \\
(6 \%), \text { H-23->LUMO (3\%), H-21->LUMO (4\%), H-19- } \\
\text { >LUMO (4\%), H-14->LUMO (3\%) }\end{array}$ \\
\hline
\end{tabular}




\begin{tabular}{|c|c|c|c|}
\hline 520.3735 & 0.025 & H-2->L+4 (41\%), H-1->L+3 (49\%) & $\mathrm{H}-3->\mathrm{L}+2(6 \%)$ \\
\hline 515.0342 & 0.576 & H-3->L+4 (68\%), H-2->L+2 (11\%) & $\begin{array}{c}\mathrm{H}-14->\mathrm{LUMO}(2 \%), \mathrm{H}-2->\mathrm{L}+3(4 \%), \mathrm{H}-1->\mathrm{L}+1(4 \%) \\
\text { HOMO->L+3 }(4 \%)\end{array}$ \\
\hline 509.3427 & 0.0025 & $\mathrm{H}-3->\mathrm{L}+3(57 \%), \mathrm{H}-1->\mathrm{L}+2(12 \%)$ & $\begin{array}{c}\mathrm{H}-34->\mathrm{LUMO}(4 \%), \mathrm{H}-4->\mathrm{L}+4(7 \%), \mathrm{H}-3->\mathrm{L}+2(3 \%), \mathrm{H}- \\
2->\mathrm{L}+1(4 \%), \mathrm{HOMO}->\mathrm{L}+4(4 \%)\end{array}$ \\
\hline 500.7035 & 0.0004 & $\begin{array}{c}\text { H-34->LUMO (43\%), H-22->LUMO (11\%), H-18- } \\
\text { >LUMO (11\%) }\end{array}$ & $\begin{array}{c}\text { H-28->LUMO (2\%), H-27->LUMO (2\%), H-17->LUMO } \\
(5 \%), \text { H-12->LUMO (2\%), H-11->LUMO (2\%), H-4->L+4 } \\
(5 \%), \text { H-3->L+3 }(5 \%)\end{array}$ \\
\hline 498.0885 & 0.0223 & $\begin{array}{c}\text { H-33->LUMO (38\%), H-21->LUMO (13\%), H-19- } \\
\text { >LUMO (24\%) }\end{array}$ & 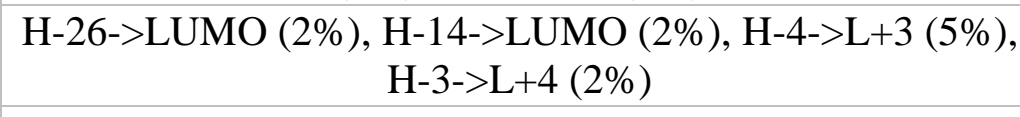 \\
\hline 492.6459 & 0.3022 & $\mathrm{H}-4->\mathrm{L}+3(77 \%)$ & H-19->LUMO (3\%), H-3->L+4 (4\%), H-2->L+2 (5\%) \\
\hline 492.4502 & 0.0528 & $\begin{array}{c}\text { H-24->LUMO (34\%), H-18->LUMO (20\%), H-16- } \\
\text { >LUMO (22\%) }\end{array}$ & H-25->LUMO (3\%), H-4->L+1 (3\%), H-2->L+4 (9\%) \\
\hline 489.0895 & 0.0365 & $\begin{array}{c}\text { H-31->LUMO (16\%), H-23->LUMO (38\%), H-20- } \\
>\text { LUMO (19\%) }\end{array}$ & $\begin{array}{c}\text { H-33->LUMO (4\%), H-21->LUMO (6\%), H-15->LUMO } \\
(2 \%), \mathrm{H}-4->\mathrm{L}+2(4 \%)\end{array}$ \\
\hline 486.6133 & 0.0046 & H-25->LUMO (58\%) & $\begin{array}{c}\text { H-34->LUMO (5\%), H-27->LUMO (2\%), H-24->LUMO } \\
(5 \%), \text { H-18->LUMO (8\%), H-17->LUMO (8\%), H-16- } \\
\text { >LUMO (3\%), H-4->L+4 (5\%) }\end{array}$ \\
\hline 485.1851 & 0.1721 & $\begin{array}{c}\mathrm{H}-23->\mathrm{LUMO}(10 \%), \mathrm{H}-4->\mathrm{L}+2(28 \%), \mathrm{H}-2->\mathrm{L}+3 \\
(17 \%)\end{array}$ & $\begin{array}{c}\text { H-31->LUMO (7\%), H-21->LUMO (3\%), H-20->LUMO } \\
(6 \%), \text { H-19->LUMO (4\%), H-15->LUMO (6\%), H-1->L+4 } \\
(8 \%)\end{array}$ \\
\hline 477.7995 & 0.0251 & $\mathrm{H}-4->\mathrm{L}+4(66 \%)$ & $\begin{array}{c}\mathrm{H}-22->\mathrm{LUMO}(3 \%), \mathrm{H}-2->\mathrm{L}+1(8 \%), \mathrm{H}-2->\mathrm{L}+4(3 \%), \mathrm{H}- \\
1->\mathrm{L}+2(7 \%)\end{array}$ \\
\hline 475.5819 & 0.0497 & $\begin{array}{c}\text { H-30->LUMO (26\%), H-26->LUMO (11\%), H-20- } \\
>\text { LUMO (28\%) }\end{array}$ & $\begin{array}{c}\text { H-39->LUMO (4\%), H-31->LUMO (7\%), H-29->LUMO } \\
(2 \%), \text { H-23->LUMO (8\%), H-4->L+2 (3\%), H-2->L+3 } \\
(2 \%)\end{array}$ \\
\hline 472.6088 & 0.6159 & $\begin{array}{c}\text { H-24->LUMO (15\%), H-4->L+1 (15\%), H-2->L+4 } \\
(19 \%), \mathrm{H}-1->\mathrm{L}+3(10 \%)\end{array}$ & $\begin{array}{c}\text { H-32->LUMO (6\%), H-28->LUMO (2\%), H-25->LUMO } \\
(8 \%), \mathrm{H}-4->\mathrm{L}+4(4 \%), \mathrm{H}-3->\mathrm{L}+2(8 \%)\end{array}$ \\
\hline 467.1245 & 0.0128 & H-31->LUMO (55\%), H-23->LUMO (13\%) & $\begin{array}{l}\text { H-30->LUMO (3\%), H-26->LUMO (2\%), H-21->LUMO } \\
(2 \%), \mathrm{H}-4->\mathrm{L}+2(7 \%), \mathrm{H}-2->\mathrm{L}+3(5 \%), \mathrm{H}-1->\mathrm{L}+4(3 \%)\end{array}$ \\
\hline
\end{tabular}


Table S8: Cartesian coordinates of the optimized (S0) geometries of the compounds 2

\begin{tabular}{|c|c|c|c|c|c|c|c|}
\hline Atom & $X$ & $\mathrm{Y}$ & $\mathrm{Z}$ & Atom & $X$ & $Y$ & Z \\
\hline $\mathrm{F}$ & -3.1149 & -1.2623 & 1.45645 & $\mathrm{C}$ & -3.2288 & -0.373 & -1.5927 \\
\hline $\mathrm{F}$ & 2.8278 & -0.7869 & -1.5153 & $\mathrm{C}$ & 8.64455 & 4.24087 & 1.59566 \\
\hline $\mathrm{F}$ & -4.6975 & -2.39 & 0.25862 & $\mathrm{H}$ & 9.33185 & 4.993 & 1.97134 \\
\hline $\mathrm{F}$ & 4.46243 & -2.2506 & -0.8951 & $\mathrm{C}$ & 1.66429 & -2.2378 & 2.7145 \\
\hline $\mathrm{N}$ & 1.68761 & -2.216 & 0.48359 & $\mathrm{H}$ & 1.91356 & -2.0344 & 3.74597 \\
\hline $\mathrm{H}$ & 1.87492 & -1.8948 & -0.4633 & $\mathrm{C}$ & 8.59226 & 2.97929 & 2.19011 \\
\hline $\mathrm{N}$ & 4.09302 & -0.3092 & 0.52017 & $\mathrm{H}$ & 9.24455 & 2.74266 & 3.02551 \\
\hline $\mathrm{N}$ & -4.066 & -0.1865 & -0.5326 & $\mathrm{C}$ & -0.5866 & -2.7447 & -2.5042 \\
\hline $\mathrm{N}$ & 5.11472 & -0.0818 & -1.7493 & $\mathrm{H}$ & 0.09955 & -3.2594 & -3.1598 \\
\hline $\mathrm{N}$ & -1.7052 & -2.1291 & -0.6819 & $\mathrm{C}$ & -6.7469 & 2.50572 & -0.3114 \\
\hline $\mathrm{H}$ & -1.9989 & -2.0074 & 0.2845 & $\mathrm{C}$ & 0.97547 & -4.8052 & -0.9844 \\
\hline $\mathrm{N}$ & -5.3764 & -0.4727 & 1.57723 & $\mathrm{H}$ & 0.45151 & -5.3782 & -1.7557 \\
\hline $\mathrm{C}$ & 5.97888 & 0.88775 & -1.2575 & $\mathrm{H}$ & 1.46566 & -5.5075 & -0.3052 \\
\hline $\mathrm{C}$ & 4.73223 & 0.92769 & 2.33426 & $\mathrm{H}$ & 1.75507 & -4.2064 & -1.4628 \\
\hline $\mathrm{H}$ & 5.24463 & 1.66344 & 2.93591 & $\mathrm{C}$ & -1.497 & -1.7376 & -2.8685 \\
\hline $\mathrm{C}$ & 3.31628 & -0.6683 & 1.5809 & $\mathrm{H}$ & -1.6577 & -1.3414 & -3.8607 \\
\hline $\mathrm{C}$ & -0.0203 & -3.9249 & -0.1918 & $\mathrm{C}$ & -6.9547 & 4.83777 & -0.9675 \\
\hline $\mathrm{C}$ & 6.86489 & 2.29389 & 0.62245 & $\mathrm{H}$ & -6.4853 & 5.78527 & -1.215 \\
\hline $\mathrm{C}$ & -0.7315 & -2.9689 & -1.1341 & $\mathrm{C}$ & -8.1489 & 2.40672 & -0.3006 \\
\hline $\mathrm{C}$ & -4.9066 & 0.89525 & -0.833 & $\mathrm{H}$ & -8.6116 & 1.45691 & -0.0536 \\
\hline $\mathrm{C}$ & 0.74659 & -3.1302 & 0.85215 & $\mathrm{C}$ & -5.7432 & -0.8842 & 2.81018 \\
\hline $\mathrm{C}$ & 3.71123 & 0.09673 & 2.72225 & $\mathrm{H}$ & -5.2783 & -1.7494 & 3.26104 \\
\hline $\mathrm{H}$ & 3.24301 & 0.04037 & 3.69383 & $\mathrm{C}$ & -4.5663 & 1.36904 & -2.1335 \\
\hline $\mathrm{C}$ & 5.92624 & 1.26613 & 0.10449 & $\mathrm{H}$ & -5.0499 & 2.19104 & -2.6395 \\
\hline $\mathrm{C}$ & 2.27186 & -1.6491 & 1.59728 & $\mathrm{C}$ & 0.71723 & -3.1654 & 2.24702 \\
\hline $\mathrm{C}$ & 4.99295 & 0.67315 & 0.95588 & $\mathrm{H}$ & 0.0847 & -3.7987 & 2.85064 \\
\hline
\end{tabular}




\begin{tabular}{|l|r|r|r|r|r|r|r|}
\hline C & 6.81378 & 1.30749 & -2.3177 & C & -8.9397 & 3.50661 & -0.6282 \\
\hline H & 7.6011 & 2.04261 & -2.2414 & H & -10.021 & 3.40972 & -0.6267 \\
\hline C & 5.38572 & -0.2578 & -3.0608 & C & 6.93057 & 3.56537 & 0.02763 \\
\hline H & 4.83117 & -0.9794 & -3.6437 & H & 6.27367 & 3.79681 & -0.8044 \\
\hline C & 6.42998 & 0.58972 & -3.4521 & C & -3.533 & 0.59457 & -2.5991 \\
\hline H & 6.85158 & 0.65834 & -4.4447 & H & -3.0108 & 0.69188 & -3.5394 \\
\hline C & -2.1981 & -1.3606 & -1.7157 & C & -1.0609 & -4.8388 & 0.49591 \\
\hline C & -6.9742 & 0.93683 & 2.35161 & H & -1.7923 & -4.2654 & 1.07176 \\
\hline H & -7.6594 & 1.7693 & 2.41166 & H & -0.5635 & -5.5411 & 1.1717 \\
\hline C & -6.1231 & 0.6539 & 1.25928 & H & -1.6044 & -5.4133 & -0.2587 \\
\hline C & -6.735 & -0.0378 & 3.32224 & B & 4.10562 & -0.9125 & -0.9247 \\
\hline H & -7.2076 & -0.1293 & 4.28972 & C & -8.346 & 4.72538 & -0.9615 \\
\hline C & -6.1604 & 3.73765 & -0.6478 & H & -8.9636 & 5.58248 & -1.2127 \\
\hline H & -5.0794 & 3.83257 & -0.6336 & C & 7.81023 & 4.53076 & 0.51452 \\
\hline C & 7.70854 & 2.0134 & 1.71033 & H & 7.84051 & 5.51226 & 0.0507 \\
\hline H & 7.68284 & 1.0268 & 2.16144 & B & -4.2996 & -1.1376 & 0.6913 \\
\hline C & -5.9078 & 1.3319 & 0.03655 & & & & \\
\hline & & & & & & & \\
\hline
\end{tabular}


Table S9: Cartesian coordinates of the optimized (S0) geometries of the compounds 1-Cu

\begin{tabular}{|c|c|c|c|c|c|c|c|}
\hline Atom & $X$ & $\mathrm{Y}$ & $\mathrm{Z}$ & Atom & $X$ & $\mathrm{Y}$ & $\mathrm{Z}$ \\
\hline $\mathrm{C}$ & 5.13397 & 2.15783 & -1.7896 & $\mathrm{C}$ & -7.1687 & -4.9975 & 3.59813 \\
\hline $\mathrm{H}$ & 4.95119 & 1.55648 & -2.6713 & $\mathrm{H}$ & -8.2433 & -5.138 & 3.66955 \\
\hline $\mathrm{C}$ & 5.84106 & 3.38251 & -1.705 & $\mathrm{C}$ & -6.3046 & -5.8271 & 4.31499 \\
\hline $\mathrm{H}$ & 6.29583 & 3.91406 & -2.5299 & $\mathrm{H}$ & -6.703 & -6.6193 & 4.942 \\
\hline $\mathrm{C}$ & 5.85964 & 3.72797 & -0.3653 & $\mathrm{C}$ & -4.9256 & -5.6339 & 4.21995 \\
\hline $\mathrm{H}$ & 6.31267 & 4.60015 & 0.08232 & $\mathrm{H}$ & -4.2456 & -6.2804 & 4.76715 \\
\hline $\mathrm{C}$ & 5.1328 & 2.71667 & 0.33533 & $\mathrm{C}$ & -4.4141 & -4.6136 & 3.41861 \\
\hline $\mathrm{C}$ & 4.83256 & 2.66795 & 1.71094 & $\mathrm{H}$ & -3.341 & -4.4723 & 3.3396 \\
\hline $\mathrm{C}$ & 5.4261 & 3.73101 & 2.58328 & $\mathrm{C}$ & -3.9284 & -1.6978 & 2.42302 \\
\hline $\mathrm{C}$ & 5.00733 & 5.06585 & 2.47869 & $\mathrm{C}$ & -3.6442 & -1.5614 & 3.82188 \\
\hline $\mathrm{H}$ & 4.23948 & 5.32672 & 1.75706 & $\mathrm{H}$ & -4.0499 & -2.1827 & 4.60663 \\
\hline $\mathrm{C}$ & 5.56353 & 6.05129 & 3.29481 & $\mathrm{C}$ & -2.7793 & -0.5041 & 3.96445 \\
\hline $\mathrm{H}$ & 5.22366 & 7.07899 & 3.20444 & $\mathrm{H}$ & -2.3563 & -0.1223 & 4.88274 \\
\hline $\mathrm{C}$ & 6.55023 & 5.71903 & 4.22424 & $\mathrm{C}$ & -2.5402 & 0.01238 & 2.65274 \\
\hline $\mathrm{H}$ & 6.98458 & 6.48687 & 4.85766 & $\mathrm{C}$ & -1.6247 & 1.07493 & 2.3565 \\
\hline $\mathrm{C}$ & 6.97612 & 4.39452 & 4.3344 & $\mathrm{C}$ & -0.7426 & 1.70934 & 3.24193 \\
\hline $\mathrm{H}$ & 7.7473 & 4.12761 & 5.0512 & $\mathrm{H}$ & -0.6524 & 1.49254 & 4.29585 \\
\hline $\mathrm{C}$ & 6.41719 & 3.40747 & 3.52226 & $\mathrm{C}$ & 0.01495 & 2.63639 & 2.51164 \\
\hline $\mathrm{H}$ & 6.7537 & 2.37867 & 3.60509 & $\mathrm{H}$ & 0.80136 & 3.2662 & 2.89756 \\
\hline $\mathrm{C}$ & 4.015 & 1.70541 & 2.33535 & $\mathrm{C}$ & -0.4102 & 2.58325 & 1.18452 \\
\hline $\mathrm{C}$ & 3.67304 & 1.64996 & 3.72719 & $\mathrm{C}$ & -0.0003 & 3.43734 & -0.0004 \\
\hline $\mathrm{H}$ & 4.03546 & 2.32585 & 4.48784 & $\mathrm{C}$ & 1.19141 & 4.34389 & 0.39559 \\
\hline $\mathrm{C}$ & 2.79551 & 0.60718 & 3.89251 & $\mathrm{H}$ & 1.48483 & 4.95476 & -0.4618 \\
\hline $\mathrm{H}$ & 2.32633 & 0.28443 & 4.81089 & $\mathrm{H}$ & 2.05902 & 3.75809 & 0.70914 \\
\hline $\mathrm{C}$ & 2.59183 & 0.03019 & 2.59882 & $\mathrm{H}$ & 0.90854 & 5.01096 & 1.21585 \\
\hline $\mathrm{C}$ & 1.6715 & -1.0348 & 2.32302 & $\mathrm{C}$ & -1.1922 & 4.34349 & -0.3965 \\
\hline $\mathrm{C}$ & 0.80425 & -1.6643 & 3.22524 & $\mathrm{H}$ & -0.9094 & 5.0105 & -1.2169 \\
\hline
\end{tabular}




\begin{tabular}{|l|r|r|r|l|r|r|r|}
\hline H & 0.73584 & -1.4479 & 4.28088 & $\mathrm{H}$ & -1.4858 & 4.95442 & 0.46078 \\
\hline $\mathrm{C}$ & 0.03417 & -2.5939 & 2.51089 & $\mathrm{H}$ & -2.0596 & 3.75738 & -0.71 \\
\hline $\mathrm{H}$ & -0.7424 & -3.2253 & 2.91335 & $\mathrm{C}$ & 0.40986 & 2.58316 & -1.1851 \\
\hline $\mathrm{C}$ & 0.43456 & -2.5451 & 1.17625 & $\mathrm{C}$ & -0.0151 & 2.6362 & -2.5123 \\
\hline $\mathrm{C}$ & 0.00005 & -3.3997 & -0.0002 & $\mathrm{H}$ & -0.8016 & 3.26584 & -2.8984 \\
\hline $\mathrm{C}$ & 1.18351 & -4.306 & -0.4197 & $\mathrm{C}$ & 0.74268 & 1.70917 & -3.2424 \\
\hline $\mathrm{H}$ & 1.49481 & -4.9175 & 0.43074 & $\mathrm{H}$ & 0.65256 & 1.49226 & -4.2963 \\
\hline $\mathrm{H}$ & 2.04625 & -3.7224 & -0.7496 & $\mathrm{C}$ & 1.62474 & 1.07494 & -2.3568 \\
\hline $\mathrm{H}$ & 0.88411 & -4.9745 & -1.2331 & $\mathrm{C}$ & 2.54026 & 0.01238 & -2.6529 \\
\hline $\mathrm{C}$ & -1.1833 & -4.3063 & 0.41915 & $\mathrm{C}$ & 2.77938 & -0.5042 & -3.9645 \\
\hline $\mathrm{H}$ & -0.8838 & -4.9747 & 1.23245 & $\mathrm{H}$ & 2.35618 & -0.1227 & -4.8828 \\
\hline $\mathrm{H}$ & -1.4944 & -4.9177 & -0.4314 & $\mathrm{C}$ & 3.64451 & -1.5613 & -3.8219 \\
\hline $\mathrm{H}$ & -2.0461 & -3.7228 & 0.74902 & $\mathrm{H}$ & 4.05028 & -2.1826 & -4.6066 \\
\hline $\mathrm{C}$ & -0.4346 & -2.545 & -1.1766 & $\mathrm{C}$ & 3.92882 & -1.6974 & -2.423 \\
\hline $\mathrm{C}$ & -0.0343 & -2.5937 & -2.5113 & $\mathrm{C}$ & 4.73698 & -2.6834 & -1.821 \\
\hline $\mathrm{H}$ & 0.74226 & -3.2251 & -2.9139 & $\mathrm{C}$ & 5.27332 & -3.7732 & -2.6904 \\
\hline $\mathrm{C}$ & -0.8045 & -1.6641 & -3.2255 & $\mathrm{C}$ & 4.41526 & -4.6134 & -3.4182 \\
\hline $\mathrm{H}$ & -0.7361 & -1.4475 & -4.2811 & $\mathrm{H}$ & 3.34207 & -4.4726 & -3.339 \\
\hline $\mathrm{C}$ & -1.6717 & -1.0346 & -2.3231 & $\mathrm{C}$ & 4.92703 & -5.6336 & -4.2196 \\
\hline $\mathrm{C}$ & -2.592 & 0.03034 & -2.5988 & $\mathrm{H}$ & 4.24714 & -6.2804 & -4.7666 \\
\hline $\mathrm{C}$ & -2.7959 & 0.60735 & -3.8924 & $\mathrm{C}$ & 6.30609 & -5.8262 & -4.3149 \\
\hline $\mathrm{H}$ & -2.3267 & 0.28471 & -4.8109 & $\mathrm{H}$ & 6.70465 & -6.6183 & -4.9419 \\
\hline $\mathrm{C}$ & -3.6736 & 1.64997 & -3.727 & $\mathrm{C}$ & 7.16998 & -4.9962 & -3.5982 \\
\hline $\mathrm{H}$ & -4.0362 & 2.3258 & -4.4876 & $\mathrm{H}$ & 8.24465 & -5.1361 & -3.6699 \\
\hline $\mathrm{C}$ & -4.0155 & 1.70529 & -2.3352 & $\mathrm{C}$ & 6.65892 & -3.983 & -2.7881 \\
\hline $\mathrm{C}$ & -4.8332 & 2.66763 & -1.7106 & $\mathrm{H}$ & 7.33087 & -3.3375 & -2.232 \\
\hline $\mathrm{C}$ & -5.4268 & 3.73075 & -2.5828 & $\mathrm{C}$ & 5.07778 & -2.7256 & -0.4532 \\
\hline $\mathrm{C}$ & -5.008 & 5.06557 & -2.4783 & $\mathrm{C}$ & 5.68006 & -3.8059 & 0.26065 \\
\hline $\mathrm{H}$ & -4.2401 & 5.32644 & -1.7567 & $\mathrm{H}$ & 5.98812 & -4.7476 & -0.1696 \\
\hline $\mathrm{C}$ & -5.5643 & 6.051 & -3.2944 & $\mathrm{C}$ & 5.73384 & -3.4294 & 1.59202 \\
\hline & & & & & & & \\
\hline
\end{tabular}




\begin{tabular}{|l|r|r|r|l|r|r|r|}
\hline $\mathrm{H}$ & -5.2244 & 7.0787 & -3.204 & $\mathrm{H}$ & 6.13044 & -3.9974 & 2.42264 \\
\hline $\mathrm{C}$ & -6.5511 & 5.71874 & -4.2237 & $\mathrm{C}$ & 5.18051 & -2.1282 & 1.65924 \\
\hline $\mathrm{H}$ & -6.9855 & 6.48658 & -4.8571 & $\mathrm{H}$ & 5.08047 & -1.4925 & 2.52976 \\
\hline $\mathrm{C}$ & -6.977 & 4.39423 & -4.3338 & $\mathrm{Cu}$ & 4.01077 & 0.00894 & -0.0521 \\
\hline $\mathrm{H}$ & -7.7483 & 4.12733 & -5.0505 & $\mathrm{Cu}$ & -4.011 & 0.00854 & 0.05209 \\
\hline $\mathrm{C}$ & -6.418 & 3.40719 & -3.5217 & $\mathrm{~N}$ & 4.71724 & 1.7496 & -0.5861 \\
\hline $\mathrm{H}$ & -6.7545 & 2.3784 & -3.6044 & $\mathrm{~N}$ & 3.3349 & 0.68778 & 1.66652 \\
\hline $\mathrm{C}$ & -5.1334 & 2.71615 & -0.335 & $\mathrm{~N}$ & 1.42099 & -1.5935 & 1.08168 \\
\hline $\mathrm{C}$ & -5.8601 & 3.72746 & 0.36574 & $\mathrm{H}$ & 1.93006 & -1.3654 & 0.23404 \\
\hline $\mathrm{H}$ & -6.313 & 4.59977 & -0.0818 & $\mathrm{~N}$ & -1.421 & -1.5934 & -1.0818 \\
\hline $\mathrm{C}$ & -5.8416 & 3.38181 & 1.70543 & $\mathrm{H}$ & -1.9302 & -1.3656 & -0.2342 \\
\hline $\mathrm{H}$ & -6.2963 & 3.91333 & 2.53036 & $\mathrm{~N}$ & -3.3351 & 0.68774 & -1.6664 \\
\hline $\mathrm{C}$ & -5.1347 & 2.15703 & 1.7899 & $\mathrm{~N}$ & -4.718 & 1.74887 & 0.58632 \\
\hline $\mathrm{H}$ & -4.952 & 1.55552 & 2.67149 & $\mathrm{~N}$ & -4.7918 & -1.7001 & -0.4526 \\
\hline $\mathrm{C}$ & -5.1796 & -2.1292 & -1.6593 & $\mathrm{~N}$ & -3.2489 & -0.6962 & 1.72686 \\
\hline $\mathrm{H}$ & -5.0795 & -1.4937 & -2.5299 & $\mathrm{~N}$ & -1.3952 & 1.62827 & 1.1095 \\
\hline $\mathrm{C}$ & -5.7326 & -3.4306 & -1.592 & $\mathrm{H}$ & -1.9327 & 1.40891 & 0.27811 \\
\hline $\mathrm{H}$ & -6.1288 & -3.9988 & -2.4226 & $\mathrm{~N}$ & 1.39484 & 1.62819 & -1.1099 \\
\hline $\mathrm{C}$ & -5.679 & -3.8069 & -0.2606 & $\mathrm{H}$ & 1.93244 & 1.40921 & -0.2785 \\
\hline $\mathrm{H}$ & -5.9868 & -4.7486 & 0.16975 & $\mathrm{~N}$ & 3.24918 & -0.6959 & -1.727 \\
\hline $\mathrm{C}$ & -5.0771 & -2.7263 & 0.45324 & $\mathrm{~N}$ & 4.7924 & -1.6993 & 0.45253 \\
\hline $\mathrm{C}$ & -4.7363 & -2.684 & 1.82102 & & & & \\
\hline $\mathrm{C}$ & -5.2724 & -3.7738 & 2.69055 & & & & \\
\hline $\mathrm{C}$ & -6.6579 & -3.9842 & 2.78802 & & & & \\
\hline $\mathrm{H}$ & -7.3301 & -3.339 & 2.23173 & & & & \\
\hline & & & & & & & \\
\hline
\end{tabular}




\section{Reference}

(1) Treutler, O.; Ahlrichs, R. Efficient molecular numerical integration schemes. J. Chem. Phys. 1995, 102, 346-354.

(2) Becke, A. D. A new mixing of Hartree-Fock and local density-functional theories. J. Chem. Phys. 1993, 98 1372-1377.

(3) Hehre, W. J. Ab initio molecular orbital theory. Acc. Chem. Res. 1976, 9, 399-406.

(4) Bauernschmitt, R.; Ahlrichs, R. Treatment of electronic excitations within the adiabatic approximation of time dependent density functional theory. Chem. Phys. Lett. 1996, 256, 454-464.

(5) Gabe, Y.; Ueno, T.; Urano, Y.; Kojima, H.; Nagano, T. Tunable design strategy for fluorescence probes based on 4-substituted BODIPY chromophore: improvement of highly sensitive fluorescence probe for nitric oxide. Anal. Bioanal. Chem. 2006, 386, 621-626.

(6) Furche, F.; Ahlrichs, R. Adiabatic time-dependent density functional methods for excited state properties. J. Chem. Phys. 2002, 117, 74337447.

(7) Scalmani, G.; Frisch, M. J.; Mennucci, B.; Tomasi, J.; Cammi, R.; Barone, V. Geometries and properties of excited states in the gas phase and in solution: Theory and application of a time-dependent density functional theory polarizable continuum model. J. Chem. Phys. 2006, 124, 94107-94115.

(8) Cossi, M.; Barone, V.; Cammi, R.; Tomasi, Ab initio study of solvated molecules: a new implementation of the polarizable continuum model. J. Chem. Phys. Lett. 1996, 255, 327-335.

(9) Tomasi, J.; Mennucci, B.; Cammi, R. Quantum Mechanical Continuum Solvation Models. Chem. Rev. 2005, 105, $2999-3094$. 